\title{
Rigorous bounds on the nonlinear saturation of instabilities to parallel shear flows
}

Article

Published Version

Shepherd, T. G. (1988) Rigorous bounds on the nonlinear saturation of instabilities to parallel shear flows. Journal Of Fluid Mechanics, 196. pp. 291-322. ISSN 0022-1120 doi: https://doi.org/10.1017/S002211208800271X Available at https://centaur.reading.ac.uk/32907/

It is advisable to refer to the publisher's version if you intend to cite from the work. See Guidance on citing.

Published version at: http://dx.doi.org/10.1017/S002211208800271X

To link to this article DOI: http://dx.doi.org/10.1017/S002211208800271X

Publisher: Cambridge University Press

All outputs in CentAUR are protected by Intellectual Property Rights law, including copyright law. Copyright and IPR is retained by the creators or other copyright holders. Terms and conditions for use of this material are defined in the End User Agreement.

www.reading.ac.uk/centaur

\section{CentAUR}


Central Archive at the University of Reading

Reading's research outputs online 


\title{
Rigorous bounds on the nonlinear saturation of instabilities to parallel shear flows $\dagger$
}

\author{
By THEODORE G. SHEPHER D $\ddagger$ \\ Department of Applied Mathematics and Theoretical Physics, University of Cambridge, \\ Silver Street, Cambridge CB3 9EW, UK
}

(Received 16 November 1987)

A novel method is presented for obtaining rigorous upper bounds on the finiteamplitude growth of instabilities to parallel shear flows on the beta-plane. The method relies on the existence of finite-amplitude Liapunov (normed) stability theorems, due to Arnol'd, which are nonlinear generalizations of the classical stability theorems of Rayleigh and Fjørtoft. Briefly, the idea is to use the finite-amplitude stability theorems to constrain the evolution of unstable flows in terms of their proximity to a stable flow. Two classes of general bounds are derived, and various examples are considered. It is also shown that, for a certain kind of forced-dissipative problem with dissipation proportional to vorticity, the finite-amplitude stability theorems (which were originally derived for inviscid, unforced flow) remain valid (though they are no longer strictly Liapunov); the saturation bounds therefore continue to hold under these conditions.

\section{Introduction}

In any discussion of the stability of a shear flow, the discovery of exponentially growing linearized normal-mode disturbances inevitably raises the question of finiteamplitude equilibration of the instability. In particular one seeks a determination of the maximum amplitude that can be attained by the growing disturbance, something that is intrinsically outside the province of linear theory.

One method for attacking this problem is provided by weakly nonlinear theory, along the lines of the now-classical work of Stuart (1960) and Watson (1960). Recent applications of such theory to zonal (i.e. parallel, $x$-invariant) shear flows on a betaplane are provided by Burns \& Maslowe (1983), Churilov \& Shukhman (1987), and Shukhman (1987). Although this approach can provide detailed information on the flow evolution, it suffers from a severe handicap in that it is restricted to instabilities that are only weakly supercritical; and because the perturbation expansions that are employed are invariably asymptotic, its range of validity must be deduced $a$ posteriori by numerical calculation. Moreover, such theories implicitly enforce modal truncations by their choice of initial conditions, thus precluding the irreversible spectral cascades that will generally occur in a system with an infinite number of degrees of freedom.

In the limit of very large supercriticality, of course, one might expect the instability to lead to a complete breakup of the flow into homogeneous, isotropic turbulence (subject to any constraint that might exist on the total zonal momentum

$\dagger$ With an appendix by P. H. Haynes.

\$ Present address: Department of Physics, University of Toronto, Toronto M5S 1A7, Canada. 
- or, more usefully, Kelvin's impulse - of the system). Such an expectation is based on an explicit presumption of randomization or dynamical ergodicity (Kraichnan 1975; Carnevale 1982). However, it has recently been shown (Shepherd 1987) that two-dimensional flow on a beta-plane is provably non-ergodic for sufficiently small wave steepness, and that the arguments of turbulence theory must therefore be used with caution in this context. In particular, one is led to ask: under what range of supercriticality can turbulent breakup be strictly precluded?

In this paper, a new method is presented for obtaining rigorous bounds on the finite-amplitude growth of instabilities to zonal shear flows. Such bounds implicitly address the two questions of nonlinear saturation and the possibility of turbulent breakup. The method relies on the existence of nonlinear Liapunov (normed) stability theorems constraining the evolution of disturbances to stable shear flows (Arnol'd 1966), and is briefly described as follows. Given a wavy (i.e. non-zonal) perturbation to an unstable zonal flow, construct a class of (nearby) stable basic zonal flows; the total disturbance to such a basic flow, consisting initially of the wavy perturbation plus a zonal-mean component, will then be constrained by the nonlinear stability theorem. By minimizing the resulting bound over the class of stable basic flows, a rigorous upper bound on the amplitude of the wavy part of the flow is obtained. However, there always remains the possibility that the bound could be tightened by considering a wider class of stable basic flows.

The plan of the paper is as follows. The governing equations are reviewed in $\S 2$, together with the two relevant nonlinear stability theorems (finite-amplitude generalizations of the Rayleigh (1880) and Fjørtoft (1950) theorems), and the method is presented formally in $\$ 3$. In $\$ 4$ it is shown that the stability theorems apply not only to inviscid flow, but also to a special kind of forced-dissipative flow where the dissipation is proportional to the vorticity, and that the saturation bounds therefore extend to this case. Two general applications of the method are given: to supercritical zonal flows on the beta-plane (\$5), using the generalized Rayleigh theorem; and to a class of almost-antisymmetric flows $(\$ 6)$, using the generalized Fjørtoft theorem. Various examples for the beta-plane are then presented in $\$ 7$, including: (a) the Bickley jet, $U=-\operatorname{sech}^{2} y ;(b)$ the free shear layer, $U=\tanh y ;(c)$ the point jet, $U=|y|$; and $(d)$ a strip of constant relative vorticity. Finally, the results are discussed in $\$ 8$.

Although the nonlinear stability theorems presented in $\$ 2$ formally break down for the case of piecewise-constant distributions of vorticity, the finite-amplitude conservation laws on which such theorems rely involve integrals of the basic-state vorticity $Q(y)$ (rather than derivatives, as in their linearized versions), and thus remain well defined. The application of Arnol'd's method (for deriving nonlinear stability theorems) in such circumstances is considered in Appendix A.

\section{Nonlinear stability theorems for disturbances to zonal flows}

The system under consideration is barotropic flow on a beta-plane, which is governed by conservation of the absolute vorticity $P \equiv \nabla^{2} \Phi+\beta y$ :

$$
\frac{\mathrm{D} P}{\mathrm{D} t} \equiv P_{t}+J(\Phi, P) \equiv P_{t}+\Phi_{x} P_{y}-\Phi_{y} P_{x}=0,
$$

where $\Phi$ is the flow stream function, $t$ the time, $x$ the zonal coordinate and $y$ the meridional coordinate, subscripts denoting partial derivatives. In $\S 4$ it will be shown 
that the results obtained below in fact hold for a certain kind of forced-dissipative flow, with the right-hand side of $(2,1)$ non-zero; but for the present we restrict attention to the conservative case. The flow is presumed to be zonally homogeneous, in the sense that the zonal average $\bar{f}$ of any quantity $f$, viz.

$$
\bar{f} \equiv \lim _{X \rightarrow \infty} \frac{1}{2 X} \int_{-X}^{+X} f(x) \mathrm{d} x,
$$

is well defined. The meridional geometry is taken to be either infinite, with boundary conditions

$$
\Phi_{y} \rightarrow \text { constant as }|y| \rightarrow \infty
$$

or bounded (a 'zonal channel' geometry), in which case the boundary conditions are

$$
\Phi_{x}=0, \quad \bar{\Phi}_{y t}=0 \quad \text { at } y=Y_{1}, Y_{2} .
$$

Now consider an $x$-invariant 'basic state' $\Phi=\Psi, P=Q$; since the advection term $J(\Psi, Q)$ vanishes identically, it follows that $\Psi, Q$ is itself a steady solution to (2.1). Defining the 'disturbance' $\psi, q$ in the natural way

$$
\Phi \equiv \Psi+\psi, \quad P \equiv Q+q,
$$

with $q \equiv \nabla^{2} \psi$, the governing equation (2.1) may then be written in the form

$$
\frac{\mathrm{D} q}{\mathrm{D} t} \equiv q_{t}+J(\Psi, q)+J(\psi, q)=-J(\psi, Q)=-\psi_{x} Q_{y}=-\frac{\mathrm{D} Q}{\mathrm{D} t}
$$

the boundary conditions $(2.2 a)$ or $(2.2 b)$ now apply to $\psi$.

Because the geometry of the problem, and the basic state, have both a spatial (zonal) and a temporal symmetry, it follows that conservation laws exist for suitable measures of a pseudomomentum and a pseudoenergy (see McIntyre \& Shepherd $1987, \S 7$ ). These conservation laws respectively lead to the following nonlinear stability theorems.

\subsection{The generalized Rayleigh theorem}

If the basic flow is presumed to have monotonic $Q(y)$, Rayleigh's (1880) theorem implies that the flow is stable. But this stability is not just linearized stability to infinitesimal normal-mode disturbances; it is in fact nonlinear stability, in the Liapunov sense that a disturbance norm at any time $t$ is bounded in terms of its value at $t=0$ (Arnol'd 1966). In particular,

$$
\int \overline{q^{2}(x, y, t)} \mathrm{d} y \leqslant \frac{\left|Q_{y}\right|_{\max }}{\left|Q_{y}\right|_{\min }} \int \overline{q^{2}(x, y, 0)} \mathrm{d} y,
$$

where the disturbance norm is here taken to be the square root of (twice) the disturbance enstrophy. Equation (2.5) holds for disturbances $q$ of any amplitude whatever, and may be considered a generalized Rayleigh stability theorem. $Q$ must be continuous, but $Q_{y}$ need not be (e.g. $\$ 7$ below). Although implicit in the important work of Arnol'd (1966), the bound (2.5) appears to have been stated explicitly for the first time by McIntyre \& Shepherd (1987, equation (6.28)); a concise derivation is available in Shepherd $(1987, \S 4)$. Note that in an unbounded domain it is necessary to have $\beta \neq 0$ in order to find flows with monotonic $Q(y)$.

In the case of a flow consisting of piecewise-constant distributions of vorticity, (2.5) becomes useless because $Q_{y}$ is then composed of delta-functions. Arnol'd's 

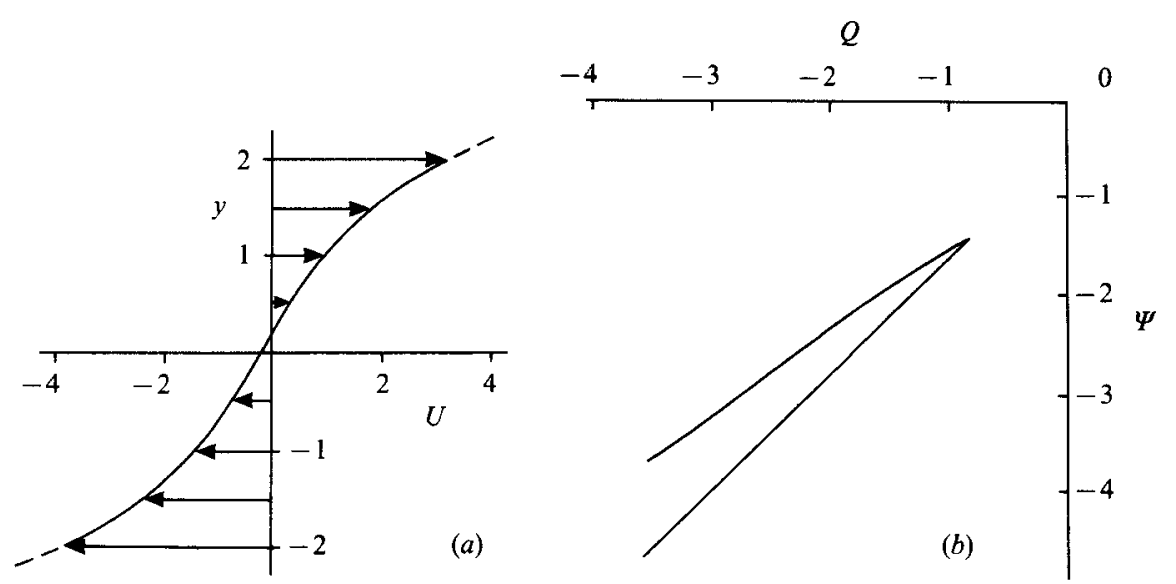

Figure 1. (a) The flow profile $U(y)$ for (2.7) with $\epsilon=0.2 . U=0$ at the single inflexion point. (b) The corresponding $\Psi(Q)$ profile, shown for the range $|y| \leqslant 2 \mathrm{~d} \Psi / \mathrm{d} Q=-U / Q_{y}$ is positive everywhere, but $\Psi(Q)$ has two branches.

method nevertheless remains perfectly valid for such flows, and leads to a stability theorem that is analogous to that above (Appendix A, $\$ \mathrm{~A} .1$ ).

\subsection{The generalized Fjortoft theorem}

If the basic flow is presumed to have $U / Q_{y} \equiv-\Psi_{y} / Q_{y}$ everywhere negative (in some frame of reference), then Fjørtoft's (1950) theorem implies stability to linearized normal-mode disturbances. But again the stability is in fact nonlinear (Arnol'd 1966), and one can show (McIntyre \& Shepherd 1987, equation (6.13); cf. also Holm et al. $1985, \S 3.3$ ) that

$$
\int \overline{\left\{|\nabla \psi|^{2}+\lambda q^{2}\right\}(x, y, t)} \mathrm{d} y \leqslant \frac{\left(-U / Q_{y}\right)_{\max }}{\left(-U / Q_{y}\right)_{\min }} \int \overline{\left\{|\nabla \psi|^{2}+\lambda q^{2}\right\}(x, y, 0)} \mathrm{d} y
$$

for any $\lambda$ in the range $\left(-U / Q_{y}\right)_{\min } \leqslant \lambda \leqslant\left(-U / Q_{y}\right)_{\max }$. Compared with $\S 2.1$, however, this case is somewhat more subtle as one must require not only that $U / Q_{y}<0$, but also that the function $\Psi(Q)$ be one-to-one. (In $\S 2.1, Q(y)$ monotonic implies trivially that $Q(y)$ is one-to-one.) To illustrate the point, the following example (with $\beta=0$ ) is instructive. Consider the basic flow

$$
U(y)=\sinh y+\epsilon \operatorname{sech} y+u_{0}, \quad Q(y)=-\cosh y+\epsilon \tanh y \operatorname{sech} y,
$$

sketched in figure 1 ( $a$ ) for $\epsilon=0.2$. The value of $u_{0}$ (which is arbitrary, because of the Galilean invariance of the problem) is chosen such that $U=0$ at the single inflexion point, so $U / Q_{y}<0$ everywhere and all linearized normal-mode disturbances must be neutral. But the $\Psi(Q)$-curve, shown in figure $1(b)$, is evidently not one-to-one, and the basic flow (2.7) is therefore not provably stable by the generalized Fjørtoft theorem. (The essential problem is that the disturbance pseudoenergy, (4.15) below, is not of definite sign in this case.) A rather similar example is discussed by McIntyre $\&$ Shepherd $(1987, \S 5)$. Only for $\epsilon=0$ do the two branches of the $\Psi(Q)$-curve collapse onto a single branch, providing a flow that is provably stable in the Liapunov sense.

A nonlinear stability theorem corresponding to (2.6) for the case of piecewiseconstant vorticity is described in Appendix A, §A.2. 


\section{A method for bounding the finite-amplitude growth of disturbances to unstable flows}

Although either of the stability theorems (2.5) and (2.6) may be used by this method, for the purpose of illustration we shall focus on the generalized Rayleigh theorem (2.5).

Suppose we are given an initial condition (at $t=0$, say) with stream function $\Phi_{0}$, consisting of a zonal-mean flow $\bar{\Phi}_{0}$ which is known to be unstable (hence with nonmonotonic $\bar{P}_{0}(y)$ ), plus a wavy component $\Phi_{0}^{\prime}$. (In this paper, the 'wavy' component of the flow is defined to be the departure from the zonal mean.) The question is: can we usefully bound the finite-amplitude growth of the wavy part of the flow? To do this, we choose to separate the total flow $\Phi$ into a basic flow $\Psi$ plus disturbance $\psi$, as in $\S 2$. The most conventional choice of a basic flow would be the initial zonal-mean flow $\bar{\Phi}_{0}$, but this would not allow the use of $(2.5)$ because $\bar{\Phi}_{0}$ is unstable by hypothesis. However, the power of the finite-amplitude theory leading to (2.5) lies in the fact that (2.5) is valid for any choice of the basic flow, even one that differs significantly from the initial zonal-mean flow. It is only the total flow $\Phi$ that is physically meaningful; the basic flow $\Psi$ is purely a mathematical device, which one is free to choose on grounds of expediency. (This would not be the case for a smallamplitude theory, by contrast.) Of course, for a given total flow $\Phi$, different choices of $\Psi$ imply different disturbances $\psi$; but if $\Psi$ is independent of $x$ (as it is taken to be here), then the wavy part of $\psi, \psi^{\prime}$, will be equal to $\Phi^{\prime}$ and will thus be independent of $\Psi$.

Therefore, introduce a family of 'basic flows' $\Psi(y ; \alpha)$ with $Q(y ; \alpha) \equiv \nabla^{2} \Psi+\beta y$ monotonic in $y$, the parameter $\alpha$ characterizing the family. With respect to any one of these basic flows, the disturbance at $t=0$ is given by

$$
\psi(x, y, 0 ; \alpha) \equiv \Phi_{0}(x, y)-\Psi(y ; \alpha)
$$

and the disturbance enstrophy at $t=0$ may be written as

$$
\left.\int \frac{1}{2} \overline{q^{2}(x, y, 0 ; \alpha)} \mathrm{d} y=\int \frac{1}{2} \overline{\left(\nabla^{2} \Phi_{0}^{\prime}\right)^{2}}+\left(\bar{\Phi}_{0 y y}-\Psi_{y y}(\alpha)\right)^{2}\right\} \mathrm{d} y
$$

Now, because the basic flow is purely zonal, it follows (by the orthogonality of zonalmean and wavy flow components) that the wavy enstrophy at any time $t \geqslant 0$ must be bounded by the disturbance enstrophy at that time, for any $\alpha$, viz.

$$
\int \frac{1}{2} \overline{\left(\nabla^{2} \Phi^{\prime}\right)^{2}(x, y, t)} \mathrm{d} y \leqslant \int \frac{1}{2} \overline{q^{2}(x, y, t ; \alpha)} \mathrm{d} y .
$$

Finally, because the basic flow is stable by hypothesis, one may invoke the Rayleigh theorem (2.5); then using (2.5), (3.2) and (3.3), one obtains

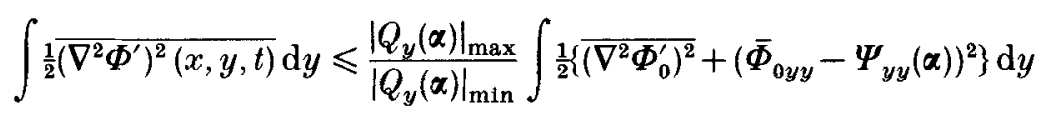

(the min and the max being taken over $y$, and yielding functions of $\alpha$ ), which represents a family of bounds on the enstrophy of the wavy part of the flow. The tightest bound (for this family) is obtained by minimizing the right-hand side of (3.4) over all $\alpha$. Of course, a better choice of basic flows might well yield an even tighter bound, but that would not invalidate (3.4). 
Whether the bound derived in this way is useful depends on whether it gives any more information than is available from the global invariants of the problem. For example, the wavy enstrophy is clearly bounded by the total relative enstrophy, which (in the inviscid problem) is constant in time; $\dagger$ hence

$$
\int \frac{1}{2} \overline{\left(\nabla^{2} \Phi^{\prime}\right)^{2}(x, y, t)} \mathrm{d} y \leqslant \int \frac{1}{2} \overline{\left(\nabla^{2} \Phi\right)^{2}(x, y, t)} \mathrm{d} y=\int \frac{1}{2} \overline{\left(\nabla^{2} \Phi_{0}\right)^{2}(x, y)} \mathrm{d} y
$$

If the boundary conditions are such that the zonal-mean flow could (in principle) become constant, then one might anticipate on global grounds that (3.5) would approach an equality. Thus (3.4) would be useful provided that the minimum of the right-hand side were less than the right-hand side of (3.5), in the sense that it would then preclude a breakup into homogeneous turbulence. If, on the other hand, the boundary conditions do not allow the zonal-mean relative vorticity to vanish, then the right-hand side of (3.5) could be replaced by something smaller, and (3.4) would have to be compared with that.

Equation (3.4) provides a bound on the wavy enstrophy. In a confined domain it would be possible to convert this into a bound on the wavy energy, by using a Poincaré inequality (see e.g. McIntyre \& Shepherd 1987, p. 541).

The procedure using the generalized Fjørtoft theorem (2.6) is entirely analogous to that described above, and yields a bound on the non-zonal part of a linear combination of the energy and enstrophy. One must, however, take heed of the discussion at the end of $\S 2.2$, and ensure that $\Psi(Q ; \alpha)$ is one-to-one for each $\alpha$.

It is natural to ask whether there are any implicit restrictions that must be placed on the choice of the family of basic flows. One might, for example, argue that the basic flow should not contain any values of vorticity not already present in the total flow (this is generally a moot point for unbounded domains). But for the bounds to hold the only formal requirement is that the stability theorems (2.5) or (2.6) hold, and these depend on the fact that the divergence of the flux of pseudomomentum or of pseudoenergy, respectively, vanish when integrated over the domain. This latter property is however assured, for any steady $x$-invariant basic flow, by the boundary conditions $(2.2)$.

\section{The forced-dissipative case}

In this section it is shown that the generalized Rayleigh theorem (2.5) and the generalized Fjørtoft theorem (2.6), derived for conservative flow, hold also for a particular kind of forced-dissipative problem (though they are no longer strictly Liapunov). It then follows that bounds of the type obtained in $\$ 3$ remain valid. Rather than (2.1), the governing equation is taken to be

$$
\frac{\mathrm{D} P}{\mathrm{D} t}=-r \nabla^{2} \Phi+F
$$

( $r$ a positive constant), with dissipation proportional to vorticity, and a constant forcing $F$ to be specified. The problem considered in $\S 3$ is the nonlinear saturation of an instability to some given zonal flow $\bar{\Phi}_{0}$; normally for such a problem to be

$\dagger$ The invariance of the absolute enstrophy $\int \frac{1}{2} \overline{P^{2}} \mathrm{~d} y$ is obvious from (2.1) and (2.2); the fact that the relative enstrophy $\int \frac{1}{2}\left(\bar{\nabla}^{2} \Phi\right)^{2} \mathrm{~d} y$ is also invariant is a consequence of the zonal symmetry of the geometry (see e.g. Shepherd 1987, §3). 
meaningful $\bar{\Phi}_{0}$ must be a steady solution of (4.1), in which case $F$ must necessarily be given by

$$
F=r \nabla^{2} \bar{\Phi}_{0}=r \bar{\Phi}_{0 y y} .
$$

The governing equation (4.1) may then be written as

$$
\frac{\mathrm{D} q}{\mathrm{D} t}=-\psi_{x} Q_{y}-r q-r\left(\Psi_{y y}-\bar{\Phi}_{0 y y}\right)=-\psi_{x} Q_{y}-r\left(q-q_{\mathrm{e}}\right)
$$

where $q_{\mathrm{e}} \equiv \bar{\Phi}_{0 y y}-\Psi_{y y}$ is the disturbance vorticity at the unstable equilibrium.

\subsection{The generalized Rayleigh theorem}

To show that (2.5) holds for the system (4.1), (4.2), equivalently (4.3), it is necessary to return to the pseudomomentum conservation law from which (2.5) was derived (e.g. Shepherd $1987, \S 4$ ). The pseudomomentum density is given by

$$
A(Q, q) \equiv-\int_{0}^{q}\left\{Y_{0}(Q+\tilde{q})-Y_{0}(Q)\right\} \mathrm{d} \tilde{q}
$$

where $Y_{0}(Q)$ is the inverse of the basic-state profile $Q(y)$; note that since $Q(y)$ is here presumed monotonic, $Y_{0}(Q)$ is also monotonic and $A(Q, q)$ is of definite sign. Following the derivation of Shepherd $(1987, \S 4)$ it may easily be verified, using $(4.3)$, that the inviscid conservation law

$$
\frac{\mathrm{d}}{\mathrm{d} t} \int \overline{A(Q, q)} \mathrm{d} y=0
$$

becomes instead

$$
\frac{\mathrm{d}}{\mathrm{d} t} \int \overline{A(Q, q)} \mathrm{d} y=r \int \overline{\left\{Y_{0}(Q+q)-Y_{0}(Q)\right\}\left(q-q_{\mathrm{e}}\right)} \mathrm{d} y .
$$

(This is in fact a special case of the more general conservation relation for forceddissipative flow recently worked out by Haynes 1988.) Now, the proof of (2.5) in the inviscid case took the form

$$
\frac{\int \frac{1}{2} \overline{q^{2}(t)} \mathrm{d} y}{\left|Q_{y}\right|_{\max }} \leqslant \int|\overline{A(Q, q(t))}| \mathrm{d} y=\int|\overline{A(Q, q(0))}| \mathrm{d} y \leqslant \frac{\int \frac{1}{2} \overline{q^{2}(0)} \mathrm{d} y}{\left|Q_{y}\right|_{\text {min }}},
$$

and it is evident that $(2.5)$ remains valid if the equality can be replaced with an inequality.

We now proceed to demonstrate that, indeed,

$$
\int|\overline{A(Q, q(t))}| \mathrm{d} y \leqslant \int|\overline{A(Q, q(0))}| \mathrm{d} y
$$

for the system (4.3), whence (2.5) holds. Without loss of generality, take $Q_{y}>0$ so that $A(Q, q) \leqslant 0$ everywhere. Now consider

$$
\begin{aligned}
\int\left\{|\overline{A(Q, q)}|-\left|\overline{A\left(Q, q_{\mathrm{e}}\right)}\right|\right\} \mathrm{d} y=\int_{D_{+}}\{|\overline{A(Q, q)}| & \left.-\left|\overline{A\left(Q, q_{\mathrm{e}}\right)}\right|\right\} \mathrm{d} y \\
& -\int_{D_{-}}\left\{\left|\overline{A\left(Q, q_{\mathrm{e}}\right)}\right|-|\overline{A(Q, q)}|\right\} \mathrm{d} y,
\end{aligned}
$$




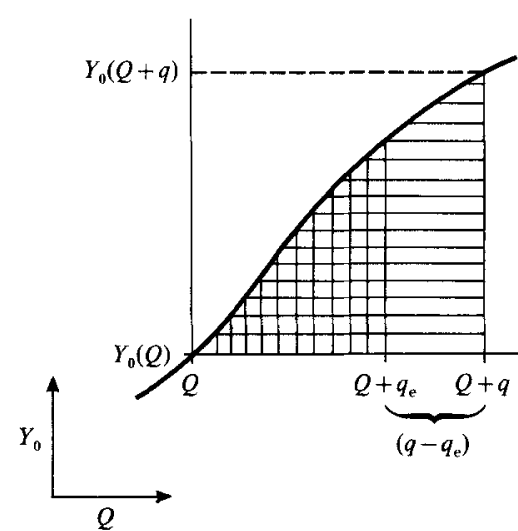

(a)

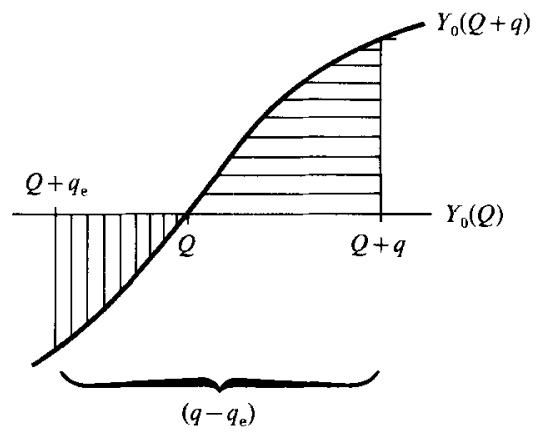

(b)

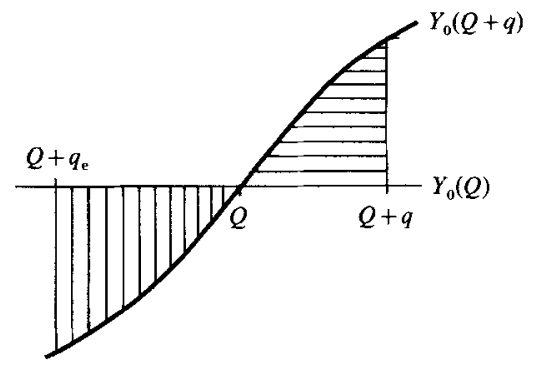

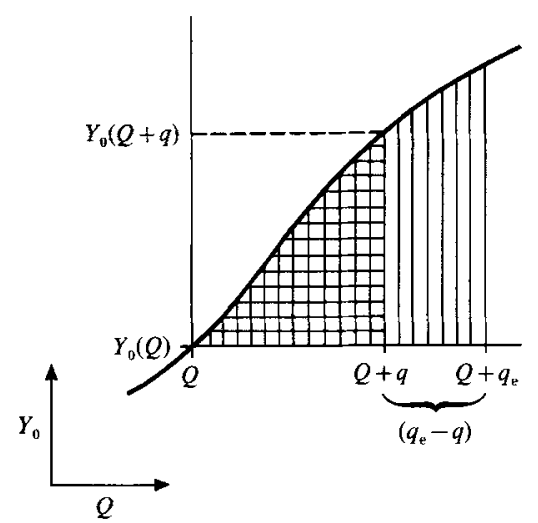

Figure 2. (a) Graphical proof of (4.9), for the two cases where $q / q_{\mathrm{e}}$ is positive (left) and negative (right). $|A(Q, q)|$ equals the area of the horizontally hatched region, and $\left|A\left(Q, q_{\mathrm{e}}\right)\right|$ the area of the vertically hatched region. Their difference (which is positive, by hypothesis) is evidently less than $\left\{Y_{0}(Q+q)-Y_{0}(Q)\right\}\left(q-q_{\mathrm{e}}\right)$. (b) As $(a)$ but for (4.10). Note that in the case of negative $q / q_{\mathrm{e}}$, the righthand side of $(4.10)$ is actually negative while the left-hand side is positive, so the inequality is satisfied trivially.

where the sub-domains $D_{+}$and $D_{-}$are those for which, at any given time $t$, the difference $|A(Q, q)|-\left|A\left(Q, q_{\mathrm{e}}\right)\right|$ respectively takes positive and negative values. In $D_{+}$, it is easy to see graphically (figure $2 a$ ) that

$$
|A(Q, q)|-\left|A\left(Q, q_{\mathrm{e}}\right)\right| \leqslant\left\{Y_{\mathbf{0}}(Q+q)-Y_{\mathbf{0}}(Q)\right\}\left(q-q_{\mathrm{e}}\right),
$$

and in $D_{-}$it is likewise evident (figure $2 b$ ) that

$$
\left|A\left(Q, q_{\mathrm{e}}\right)\right|-|A(Q, q)| \geqslant\left\{Y_{\mathbf{0}}(Q+q)-Y_{\mathbf{0}}(Q)\right\}\left(q_{\mathrm{e}}-q\right) .
$$

Combining (4.8), (4.9) and (4.10), one obtains

$$
\int\left\{\overline{A(Q, q)}|-| \overline{A\left(Q, q_{\mathrm{e}}\right)} \mid\right\} \mathrm{d} y \leqslant \int \overline{\left\{Y_{0}(Q+q)-Y_{0}(Q)\right\}\left(q-q_{\mathrm{e}}\right)} \mathrm{d} y .
$$

It therefore follows that whenever

$$
\int \overline{\mid A(Q, q)}\left|\mathrm{d} y \geqslant \int\right| \overline{A\left(Q, q_{\mathrm{e}}\right)} \mid \mathrm{d} y
$$


the right-hand side of (4.11) must be positive, from which (4.5) implies (since $A(Q, q) \leqslant 0$ for the case under discussion) that

$$
\frac{\mathrm{d}}{\mathrm{d} t} \int|\overline{A(Q, q)}| \mathrm{d} y<0 .
$$

To complete the proof of $(4.7)$, it remains to show that

$$
\int \overline{A(Q, q(0))}\left|\mathrm{d} y \geqslant \int\right| \overline{A\left(Q, q_{\mathrm{e}}\right)} \mid \mathrm{d} y .
$$

But since by definition $q(0)=q_{\mathrm{e}}+q_{0}^{\prime}$, with $\overline{q_{0}^{\prime}}=0$, it is obvious graphically (cf. figure 2) that (4.13) must be true: when $q_{\mathrm{e}}>0$, one has

$$
\left|A\left(Q, q_{\mathrm{e}}+q_{0}^{\prime}\right)\right|-\left|A\left(Q, q_{\mathrm{e}}\right)\right| \geqslant\left\{Y_{0}\left(Q+q_{\mathrm{e}}\right)-Y_{0}(Q)\right\} q_{0}^{\prime}
$$

whenever $q_{0}^{\prime}>0$, and

$$
\left|A\left(Q, q_{\mathrm{e}}\right)\right|-\left|A\left(Q, q_{\mathrm{e}}+q_{0}^{\prime}\right)\right| \leqslant\left\{Y_{0}\left(Q+q_{\mathrm{e}}\right)-Y_{0}(Q)\right\}\left(-q_{0}^{\prime}\right)
$$

whenever $q_{0}^{\prime}<0$, which together imply (since $\overline{q_{0}^{\prime}}=0$ ) that

$$
\left|\overline{A\left(Q, q_{\mathrm{e}}+q_{0}^{\prime}\right)}\right| \geqslant\left|\overline{A\left(Q, q_{\mathrm{e}}\right)}\right|
$$

and similarly when $q_{\mathrm{e}}<0$. We have thus established the logical chain

$$
\begin{aligned}
\int|\overline{A(Q, q(t))}| \mathrm{d} y>\int|\overline{A(Q, q(0))}| \mathrm{d} y & \Rightarrow \int|\overline{A(Q, q(t))}| \mathrm{d} y>\int\left|\overline{A\left(Q, q_{\mathrm{e}}\right)}\right| \mathrm{d} y \\
& \Rightarrow \frac{\mathrm{d}}{\mathrm{d} t} \int|\overline{A(Q, q(t))}| \mathrm{d} y<0,
\end{aligned}
$$

implying that the absolute value of the pseudomomentum can never exceed its initial value, from which (4.7) follows immediately. Therefore the generalized Rayleigh theorem (2.5) holds for the forced-dissipative system (4.1), (4.2). Note, however, that the stability theorem is no longer strictly Liapunov (in the sense that disturbances can be kept arbitrarily small), because $\int|\overline{A(Q, q(0))}| \mathrm{d} y$ will have a positive lower bound, namely $\int\left|A\left(Q, q_{\mathrm{e}}\right)\right| \mathrm{d} y$.

\subsection{The generalized Fjortoft theorem}

The proof that (2.6) holds for the system (4.1), (4.2), equivalently (4.3), is largely similar to the case of (2.5), though with one additional step. Here the relevant conservation law is that for the pseudoenergy, which is given by

$$
E(Q, q)+B(Q, q) \equiv \frac{1}{2}|\nabla \psi|^{2}+\int_{0}^{q}\{\Psi(Q+\tilde{q})-\Psi(Q)\} \mathrm{d} \tilde{q}
$$

(e.g. McIntyre \& Shepherd $1987, \S 3$ ); $†$ since $\mathrm{d} \Psi / \mathrm{d} Q$ is presumed positive, $B(Q, q)$ is likewise positive (as is $E$, of course). Following the derivation of McIntyre \& Shepherd, it may easily be verified, using (4.3), that the inviscid conservation law

$$
\frac{\mathrm{d}}{\mathrm{d} t} \int \overline{(E+B)} \mathrm{d} y=0
$$

$\dagger$ We here use $E$, rather than McIntyre $\&$ Shepherd's $\hat{E}$, purely for notational convenience and emphasize that it is not to be confused with the total energy. 
becomes instead

$$
\frac{\mathrm{d}}{\mathrm{d} t} \int \overline{(E+B)} \mathrm{d} y=r \int \overline{\psi\left(q-q_{\mathrm{e}}\right)} \mathrm{d} y-r \int \overline{\{\Psi(Q+q)-\Psi(Q)\}\left(q-q_{\mathrm{e}}\right)} \mathrm{d} y .
$$

The quantity $B(Q, q)$ is formally very similar to $|A(Q, q)|$ in $\S 4.1$, so by orthogonality of zonal and non-zonal flow components we have, analogous to (4.13), that

$$
\int\{\overline{E(Q, q(0))}+\overline{B(Q, q(0))}\} \mathrm{d} y \geqslant \int\left\{\overline{E\left(\bar{Q}, q_{\mathrm{e}}\right)}+\overline{B\left(Q, q_{\mathrm{e}}\right)}\right\} \mathrm{d} y
$$

It remains to establish that the right-hand side of (4.16) must be negative whenever

$$
\int\{\overline{E(Q, q)}+\overline{B(Q, q)}\} \mathrm{d} y \geqslant \int\left\{\overline{E\left(Q, q_{\mathrm{e}}\right)}+\overline{B\left(Q, q_{\mathrm{e}}\right)}\right\} \mathrm{d} y
$$

which (together with (4.17)) will guarantee that the pseudoenergy can never exceed its initial value, and that the generalized Fjørtoft theorem will therefore continue to hold for the system (4.1), (4.2).

To do this, consider the expression

$$
\int\left\{\overline{E(Q, q)}+\overline{B(Q, q)}-\overline{E\left(Q, q_{\mathrm{e}}\right)}-\overline{B\left(Q, q_{\mathrm{e}}\right)}\right\} \mathrm{d} y .
$$

Once again exploiting the analogy between $B(Q, q)$ and $|A(Q, q)|$, the graphical proof presented in figure 2 here implies that

$$
\int\left\{\overline{B(Q, q)}-\overline{B\left(Q, q_{\mathrm{e}}\right)}\right\} \mathrm{d} y \leqslant \int \overline{\{\Psi(Q+q)-\Psi(Q)\}\left(q-q_{\mathrm{e}}\right)} \mathrm{d} y
$$

analogous to $(4.11)$; while

$$
\int\left\{\overline{E(Q, q)}-\overline{E\left(Q, q_{\mathrm{e}}\right)}\right\} \mathrm{d} y=\int \frac{1}{2}\left\{\psi_{\mathrm{e}} q_{\mathrm{e}}-\overline{\psi q}\right\} \mathrm{d} y,
$$

after integrating by parts, and using (2.2) together with the fact that $\bar{\psi}=\psi_{\mathrm{e}}$ on the boundary. Now, employing a similar application of the boundary conditions one has

$$
\int \overline{\left(\psi-\psi_{\mathrm{e}}\right)\left(q-q_{\mathrm{e}}\right)} \mathrm{d} y=-\int\left\{\overline{\left(\left(\psi-\psi_{\mathrm{e}}\right)_{y}\right)^{2}}+\overline{\left(\left(\psi-\psi_{\mathrm{e}}\right)_{x}\right)^{2}}\right\} \mathrm{d} y<0
$$

It then follows that

$$
\begin{aligned}
& \int \frac{1}{2}\left\{\psi_{\mathrm{e}} q_{\mathrm{e}}-\overline{\psi q}\right\} \mathrm{d} y=-\int \overline{\psi\left(q-q_{\mathrm{e}}\right)} \mathrm{d} y+\frac{1}{2} \int \overline{\left(\psi_{\mathrm{e}} q-\psi q_{\mathrm{e}}\right)} \mathrm{d} y \\
& \leqslant-\int \frac{1}{2} \int \overline{\left(\psi-\psi_{\mathrm{e}}\right)\left(q-q_{\mathrm{e}}\right)} \mathrm{d} y \\
& \overline{\psi\left(q-q_{\mathrm{e}}\right)} \mathrm{d} y
\end{aligned}
$$

the equality being an identity, and the inequality arising from (4.22) together with the readily established fact that

$$
\int \overline{\left(\psi_{\mathrm{e}} q-\psi \overline{q_{\mathrm{e}}}\right)} \mathrm{d} y=0
$$


Combining $(4.20),(4.21)$ and $(4.23)$ then yields the relation

$$
(4.19) \leqslant \int\left\{\overline{\Psi(Q+q)-\bar{\Psi}(Q)\}\left(q-q_{\mathrm{e}}\right)} \mathrm{d} y-\int \overline{\psi\left(q-q_{\mathrm{e}}\right)} \mathrm{d} y .\right.
$$

From (4.24) it is clear that the right-hand side of (4.16) is negative whenever (4.19) is positive, namely whenever (4.18) is true, which completes the proof.

\section{A general bound for supercritical zonal flows}

In this section the method of $\S 3$ is used to derive general bounds on the growth of disturbances to initial zonal flows

$$
\bar{U}_{0}(y) \equiv-\frac{\mathrm{d} \bar{\Phi}_{0}}{\mathrm{~d} y}(y)=u_{0} g(y)
$$

with $u_{0}$ a positive constant and $g_{y}$ a continuous function of $y$. Such flows are unstable only if

$$
u_{0}>\frac{\beta}{C} \equiv u_{\mathrm{crit}}
$$

with $C \equiv \max \left\{g_{y y}\right\}>0$, and can therefore be called supercritical whenever (5.2) is true. It is useful to write

$$
u_{0} \equiv(1+\epsilon) u_{\mathrm{crit}}=(1+\epsilon) \frac{\beta}{C}
$$

with $\epsilon$ a measure of the relative supercriticality (not assumed small!). For flows of this type, a natural family of stable basic flows is provided by

$$
U(y ; \alpha)=(1-\alpha) \frac{\beta}{C} g(y),
$$

with $0<\alpha \leqslant 1$. The object is now to evaluate the right-hand side of (3.4), and then calculate its minimum over the range of $\alpha$.

From (5.4), one has

$$
\beta-\frac{\beta}{C}(1-\alpha) C \leqslant Q_{y}(y ; \alpha) \leqslant \beta-\frac{\beta}{C}(1-\alpha) c,
$$

where $c \equiv \min \left\{g_{y y}\right\}$, and therefore

$$
\frac{\left|Q_{y}(\alpha)\right|_{\max }}{\left|Q_{y}(\alpha)\right|_{\min }}=\frac{1-\frac{c}{C}+\alpha \frac{c}{C}}{\alpha} \equiv \frac{1}{\alpha}(1-\gamma+\alpha \gamma),
$$

where $\gamma \equiv c / C$. The initial disturbance enstrophy is given by

$$
\begin{aligned}
\int \frac{1}{2} \overline{q^{2}(0 ; \alpha)} \mathrm{d} y & =\int \frac{1}{2} \overline{\left(\nabla^{2} \Phi_{0}^{\prime}\right)^{2}} \mathrm{~d} y+\int \frac{1}{2}\left\{\frac{\beta}{C}(\alpha+\epsilon) g_{y}\right\}^{2} \mathrm{~d} y \\
& =\frac{\beta^{2} Z}{2 C^{2}}\left\{(\alpha+\epsilon)^{2}+z_{0}\right\}
\end{aligned}
$$

where

$$
Z \equiv \int\left(g_{y}\right)^{2} \mathrm{~d} y, \quad z_{0} \equiv \frac{2 C^{2}}{\beta^{2} Z} \int \frac{1}{2} \overline{\left(\nabla^{2} \Phi_{0}^{\prime}\right)^{2}} \mathrm{~d} y .
$$


Therefore the right-hand side of (3.4), providing the bound on the enstrophy of the non-zonal part of the flow, takes the form

$$
\frac{\beta^{2} Z}{2 C^{2}} \frac{1}{\alpha}(1-\gamma+\alpha \gamma)\left\{(\alpha+\epsilon)^{2}+z_{0}\right\} \equiv \frac{\beta^{2} Z}{2 C^{2}} f\left(\alpha, \epsilon, \gamma, z_{0}\right) .
$$

For given $\epsilon, \gamma$ and $z_{0}$, we seek the minimum of $f\left(\alpha, \epsilon, \gamma, z_{0}\right)$ over the interval $0<\alpha \leqslant 1$. Noting that $f$ diverges as $\alpha \rightarrow 0$, this minimum will either be a local minimum, attained at some $\alpha_{\min }<1$, or it will be attained at $\alpha=1$; but in the latter case the bound is identical to that obtained from conservation of total relative enstrophy, viz. (3.5), since the basic flow $U$ vanishes for $\alpha=1$.

Seeking the local minimum, setting $\partial f / \partial \alpha=0$ leads to the cubic equation

$$
\alpha^{3}+\left\{\frac{(1-\gamma)}{2 \gamma}+\epsilon\right\} \alpha^{2}-\frac{(1-\gamma)}{2 \gamma}\left(\epsilon^{2}+z_{0}\right)=0 .
$$

For sufficiently small $z_{0}(5.10)$ has three real roots, and the minimum of $f$ is attained at

$$
\alpha_{\min }=2 \rho \cos \left(\frac{\phi+4 \pi}{3}\right)-\rho
$$

where

$$
\rho \equiv \frac{1}{3}\left\{\frac{(1-\gamma)}{2 \gamma}+\epsilon\right\}, \quad \cos \phi \equiv \frac{27}{4}\left\{\frac{(1-\gamma)}{\gamma}\right\}\left\{\frac{(1-\gamma)}{2 \gamma}+\epsilon\right\}^{-3}\left\{\epsilon^{2}+z_{0}\right\}-1
$$

(see Bronshtein \& Semendyayev 1985, $§ 2.4 .2 .3$ ). Figure 3 shows $f\left(\alpha_{\min }, \epsilon, \gamma, z_{0}\right)$ as a function of $\epsilon$, for various values of $z_{0}$, for the two cases $\gamma=-\frac{1}{3}$ and $\gamma=-1$. The bound obtained by considering the total relative enstrophy in the flow, which may be calculated by putting $\alpha=1$ in the formula for $f$, is also drawn; so long as the former bound is less than the latter, the stability theorem can be regarded as constraining the flow behaviour. It may be seen from figure 3 that the bound obtained with (5.11) is only useful for $z_{0} \ll 1$, and that as $\gamma$ becomes more negative the useful range of $z_{0}$ decreases.

An important special case is that where the initial wavy disturbance has infinitesimal amplitude, viz. the limit $z_{0} \rightarrow 0$. In that limit the cubic (5.10) is easily seen to factor to

and

$$
\begin{gathered}
(\alpha+\epsilon)\left\{\alpha^{2}+\frac{(1-\gamma)}{2 \gamma} \alpha-\frac{(1-\gamma)}{2 \gamma} \epsilon\right\}=0, \\
\alpha_{\min }=\frac{(\gamma-1)}{4 \gamma}\left\{1-\left\{1-\frac{8 \gamma \epsilon}{(\gamma-1)}\right\}^{\frac{1}{2}}\right\} .
\end{gathered}
$$

It is evident from (5.13) that for sufficiently small $\epsilon, \alpha_{\min }$ is well approximated as $\alpha_{\min } \approx \epsilon$. In fact, numerical calculation reveals that in using $\alpha=\epsilon$ the true minimum of $f$ is overestimated by no more than about $3 \%$ over the useful range of $\epsilon$. The latter is the range for which $f\left(\alpha_{\min }, \epsilon, \gamma, 0\right)<f(1, \epsilon, \gamma, 0)$; taking $f\left(\alpha_{\min }, \epsilon, \gamma, 0\right) \approx f(\epsilon, \epsilon, \gamma, 0)$ the condition on $\epsilon$ becomes

$$
4 \epsilon(1-\gamma+\epsilon \gamma)<(1+\epsilon)^{2} \Leftrightarrow(1-4 \gamma) \epsilon^{2}-2(1-2 \gamma) \epsilon+1>0 .
$$

Under the reasonable presumption that $\gamma \equiv \min \left\{g_{y y}\right\} / \max \left\{g_{y y}\right\}$ is negative, (5.14) is satisfied for

$$
\epsilon<\frac{1}{1-4 \gamma}
$$



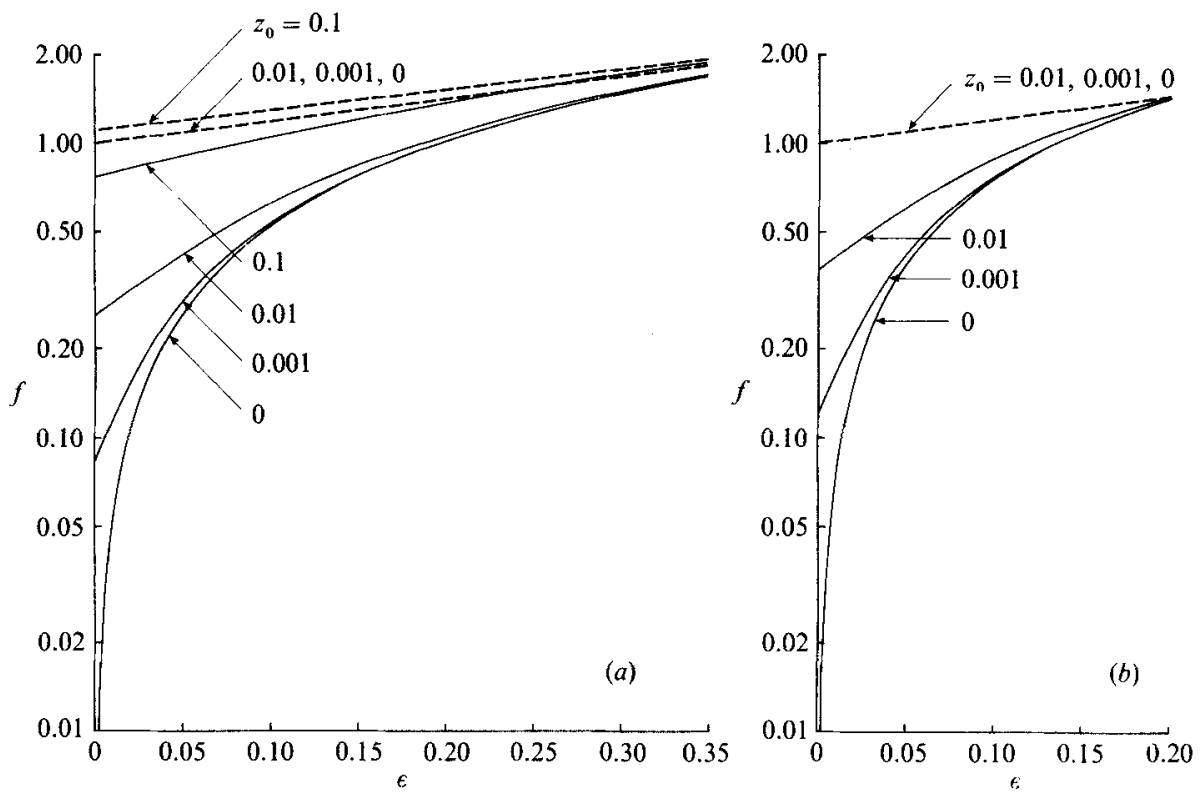

FIGURE 3. (a) The solid curves show the graph of $f\left(\alpha_{\min }, \epsilon, \gamma, z_{0}\right)$ versus $\epsilon$ for $\gamma=-\frac{1}{3}$ and for various $z_{0}$, as indicated. The upper dashed curves show $f\left(1, \epsilon, \gamma, z_{0}\right)$, which represents the value of $f$ corresponding to the total amount of relative enstrophy in the system. (b) Same as in (a), except for $\gamma=-1$.

which represents the range of $\epsilon$ (for $z_{0}=0$ ) for which a complete breakup into nonzonal motion is definitely precluded. Both $f\left(\alpha_{\min }, \epsilon, \gamma, 0\right)$ and $f(1, \epsilon, \gamma, 0)$ are shown in figure 3 for $\gamma=-\frac{1}{3}$ and $\gamma=-1$. One may therefore write down the general bound

$$
\int \frac{1}{2} \overline{\left(\nabla^{2} \Phi^{\prime}\right)^{2}} \mathrm{~d} y \leqslant \frac{\beta^{2} Z}{2 C^{2}} F(\gamma, \epsilon)
$$

valid in the limit $z_{0} \rightarrow 0$, where

$$
F(\gamma, \epsilon) \equiv\left\{\begin{array}{cc}
4 \epsilon(1-\gamma+\epsilon \gamma) & \text { for } \epsilon \leqslant(1-4 \gamma)^{-1} \\
(1+\epsilon)^{2} & \text { for } \epsilon \geqslant(1-4 \gamma)^{-1}
\end{array}\right\}
$$

Equation (5.16) represents a rigorous upper bound on the possible nonlinear growth of an infinitesimal wavy disturbance to an unstable zonal flow (5.1) with supercriticality $\epsilon$.

\section{A general bound for almost-antisymmetric flows}

In this section attention is directed to a class of zonal flows which have $U / Q_{y}<0$ everywhere (at least in some frame of reference) but for which $\Psi(Q)$ is not one-toone. (For the general case we must have $\beta=0$; but see the discussion below (6.7).) The example of (2.7) and figure 1 is a case in point. These may be considered almostantisymmetric flows, in the sense that

$$
\bar{U}_{0}(y)=\hat{U}(y)+\epsilon \tilde{U}(y), \quad \bar{P}_{0}(y)=-\hat{U}_{y}-\epsilon \tilde{U}_{y},
$$

with $\hat{U}(y)$ an odd function of $y$ having a single inflexion point (at $y=0$ ), $\hat{U} / \hat{U}_{y y}>0$, and $\epsilon$ presumed small. Provided that $\epsilon$ is sufficiently small, it will generally be the 
case that $\bar{U}_{0} / \bar{P}_{0 y}<0$ in some frame of reference, and hence linearized normal-mode disturbances must be neutral, but the flow (6.1) will not be provably stable in the Liapunov sense because of the problem of multifunctionality discussed at the end of $\S 2$. However, by construction the flow

$$
U(y)=\hat{U}(y), \quad Q(y)=-\hat{U}_{y}
$$

is provably stable, by the generalized Fjørtoft (or Arnol'd) theorem, and may serve as a basic flow. Relative to this basic flow, then, (2.6) immediately provides the following bound on the energy and enstrophy of non-zonal disturbances to (6.1):

$$
\int \frac{1}{2}\left\{\left.\overline{\mid \nabla} \Phi^{\prime}\right|^{2}+\lambda \overline{\left(\nabla^{2} \bar{\Phi}^{\prime}\right)^{2}}\right\}(t) \mathrm{d} y \leqslant \frac{\left(-U / Q_{y}\right)_{\max }}{\left(-U / Q_{y}\right)_{\min }}\left\{\zeta_{0}(\lambda)+\epsilon^{2} \int \frac{1}{2}\left\{\tilde{U}^{2}+\lambda\left(\tilde{U}_{y}\right)^{2}\right\} \mathrm{d} y\right\}
$$

for any $\lambda$ in the range $\left(-U / Q_{y}\right)_{\min } \leqslant \lambda \leqslant\left(-U / Q_{y}\right)_{\max }$, with

$$
\left.\zeta_{0}(\lambda) \equiv \int \frac{1}{2} \mid \overline{\left.\nabla \Phi_{0}^{\prime}\right|^{2}}+\lambda \overline{\left(\nabla^{2} \Phi_{0}^{\prime}\right)^{2}}\right\} \mathrm{d} y
$$

Thus, apart from $\zeta_{0}$, a flow that departs from exact antisymmetry by $O(\epsilon)$ cannot support the growth of wavy energy and enstrophy beyond $O\left(\epsilon^{2}\right)$.

As a concrete example, consider an initial zonal flow given by (2.7), taking $u_{0}=0$, in the domain $Y_{1} \leqslant y \leqslant Y_{2}$ and in the limit $\zeta_{0} \rightarrow 0$. This is of the form (6.1), with $\hat{U}(y)=\sinh y$, and taking the basic flow (6.2) it follows that

$$
\frac{U}{Q_{y}}=\frac{\sinh y}{-\sinh y}=-1
$$

so $\lambda=1$ necessarily, while

$$
\begin{aligned}
\int_{Y_{1}}^{Y_{2}} \frac{1}{2}\left\{\tilde{U}^{2}+\left(\tilde{U_{y}}\right)^{2}\right\} \mathrm{d} y & =\int_{Y_{1}}^{Y_{2}} \frac{1}{2}\left\{\operatorname{sech}^{2} y+\tanh ^{2} y \operatorname{sech}^{2} y\right\} \mathrm{d} y \\
& =\frac{1}{2}\left\{\tanh Y_{2}-\tanh Y_{1}+\frac{1}{3}\left(\tanh ^{3} Y_{2}-\tanh ^{3} Y_{1}\right)\right\}
\end{aligned}
$$

In the limit $Y_{1} \rightarrow-\infty, Y_{2} \rightarrow \infty$, the expression (6.6) approaches $\frac{4}{3}$ (this is in fact its upper limit), and using (6.5) the bound (6.3) takes the form (for $\zeta_{0} \rightarrow 0$ )

$$
\int_{Y_{1}}^{Y_{2}} \frac{1}{2}\left\{\overline{\left.\nabla \Phi^{\prime}\right|^{2}}+\overline{\left(\nabla^{2} \Phi^{\prime}\right)^{2}}\right\}(t) \mathrm{d} y \leqslant \frac{4 \epsilon^{2}}{3}
$$

It turns out that the condition $\beta=0$ can be relaxed in cases, such as the example above, where $\mu \hat{U}_{y y}=\hat{U}$ with $\mu$ a positive constant. For then one may choose a frame of reference where the basic flow is given by

$$
U(y)=\hat{U}(y)-\beta \mu, \quad Q(y)=\beta y-\hat{U}_{y}
$$

so that

$$
\frac{U}{Q_{y}}=\frac{\hat{U}-\beta \mu}{\beta-\hat{U} \mu}=-\mu
$$


and is constant, and $\lambda=\mu$ necessarily; the bound $(6.3)$ then takes the form

$$
\int \frac{1}{2}\left\{\overline{\left|\nabla \Phi^{\prime}\right|^{2}}+\mu \overline{\left(\nabla^{2} \Phi^{\prime}\right)^{2}}\right\}(t) \mathrm{d} y \leqslant \zeta_{0}(\mu)+\epsilon^{2} \int \frac{1}{2}\left\{\tilde{U}^{2}+\mu\left(\tilde{U_{y}}\right)^{2}\right\} \mathrm{d} y
$$

of which (6.7) is a special case, valid for arbitrary $\beta$ although itself independent of $\beta$.

\section{Specific examples, using the generalized Rayleigh theorem}

\subsection{The Bickley jet}

First consider the Bickley jet

$$
\bar{U}_{0}(y)=-u_{0} \operatorname{sech}^{2} y, \quad \bar{P}_{0}(y)=\beta y-2 u_{0} \operatorname{sech}^{2} y \tanh y \quad(-\infty<y<\infty),
$$

shown in figure 4. This zonal flow is of the type considered in $\$ 5$, and is known to be unstable for $u_{0}>u_{\text {crit }}=\frac{1}{2} \beta$. The parameters used in $\$ 5$ take the values

$$
C=2, \quad c=-\frac{2}{3}, \quad \gamma=-\frac{1}{3}, \quad Z=\frac{16}{15},
$$

and the enstrophy of the non-zonal part of the flow is therefore bounded by (5.9) with $\alpha$ given by (5.11); see figure 3(a) for the dependence of $f$ on $\epsilon$ and $z_{0}$. (Here $\epsilon$ is the relative supercriticality, as defined by (5.3).) In the special case of an infinitesimal initial wavy disturbance, $z_{0} \rightarrow 0$, the bound (5.16) may be invoked, yielding

$$
\int_{-\infty}^{\infty} \frac{1}{2} \overline{\left(\nabla^{2} \Phi^{\prime}\right)^{2}} \mathrm{~d} y \leqslant\left\{\begin{array}{ll}
\frac{8}{45} \beta^{2} \epsilon(4-\epsilon) & \text { for } \epsilon \leqslant 0.43 \\
\frac{2}{15} \beta^{2}(1+\epsilon)^{2} & \text { for } \epsilon \geqslant 0.43
\end{array}\right\}
$$

It turns out that this bound can be significantly tightened by considering a rather different family of stable basic states, which has discontinuous $Q_{y}$, namely

$$
Q(y ; \alpha)=\left\{\begin{array}{ll}
\alpha y & \text { for }|y| \leqslant y_{1} \\
\bar{P}_{0}(y) & \text { for }|y| \geqslant y_{1} ;
\end{array}\right\}
$$

the construction is shown in figure $4(b)$, and $y_{1}$ is the (positive) root of the equation

$$
(\beta-\alpha) y=2 u_{0} \operatorname{sech}^{2} y \tanh y
$$

In effect, the basic flow differs from the initial zonal flow only in the immediate vicinity of its unstable region. For this family,

$$
\left|Q_{y}\right|_{\text {min }}=\alpha, \quad\left|Q_{y}\right|_{\max }=\left\{\begin{array}{ll}
\bar{P}_{0}\left(y_{1}\right) & \text { if } y_{1} \geqslant y_{0} \\
\bar{P}_{0}\left(y_{0}\right)=\beta+\frac{2}{3} u_{0} & \text { if } y_{1} \leqslant y_{0},
\end{array}\right\}
$$

where $y_{0}=\operatorname{arctanh}(2 / 3)^{\frac{1}{2}} \approx 1.15$, and

$$
\int_{-\infty}^{\infty} \frac{1}{2} \overline{q^{2}(0 ; \alpha)} \mathrm{d} y=\int_{-\infty}^{\infty} \frac{1}{2} \overline{\left(\nabla^{2} \Phi_{0}^{\prime}\right)^{2}} \mathrm{~d} y+\int_{0}^{y_{1}}\left\{(\beta-\alpha) y-2 u_{0} \operatorname{sech}^{2} y \tanh y\right\}^{2} \mathrm{~d} y
$$

The second term on the right-hand side of (7.7) may be integrated exactly in terms of $y_{1}$, and $y_{1}$ may be found numerically from (7.5) using an iterative root finder. Then the minimum over $\alpha$ of the right-hand side of (3.4), using (7.6) and (7.7), can be determined numerically for given

$$
\hat{z}_{0} \equiv \int \frac{1}{2}\left(\overline{\left.\nabla^{2} \Phi_{0}^{\prime}\right)^{2}} \mathrm{~d} y\right.
$$



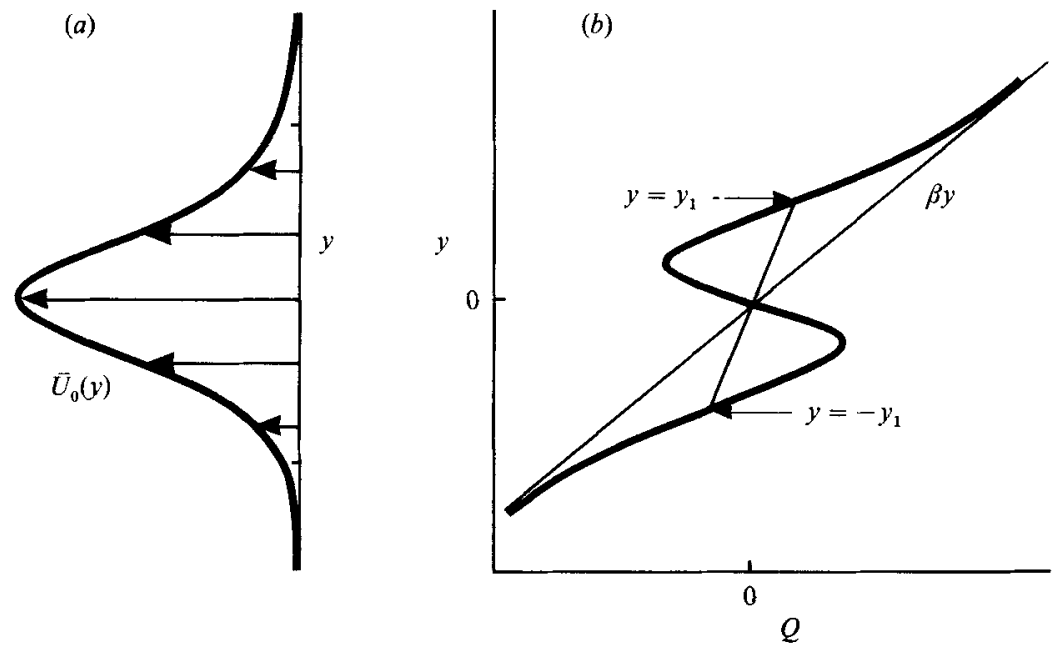

Figure 4. (a) The flow profile $\bar{U}_{0}(y)$ for the Bickley jet (7.1). (b) The corresponding $\bar{P}_{\mathbf{0}}(y)$ profile, together with the basic flow (7.4) (thin line). For notation see text.

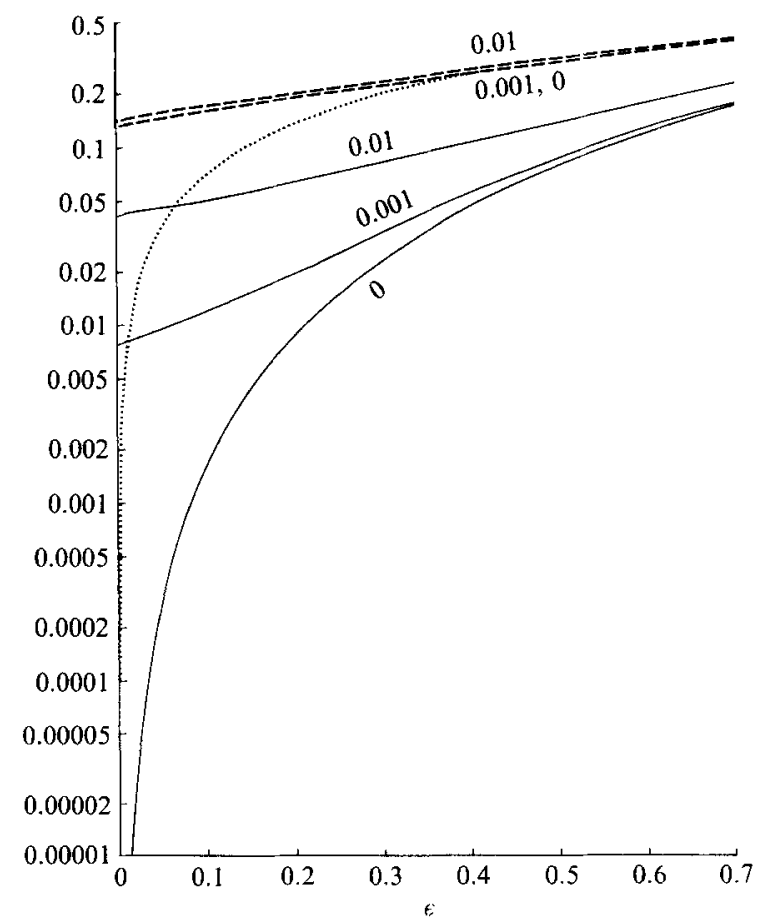

Figure 5. The bound on the wavy enstrophy for the Bickley jet, as a function of the supercriticality $\epsilon$, for $\beta=1$. The dotted curve shows (7.3), relevant to the limit $z_{0} \rightarrow 0$. The solid curves indicate the minimum over $\alpha$ of (3.4), using (7.6) and (7.7), for various $\hat{z}_{0}$; the number denotes the value of $\hat{z}_{0} / \beta^{2}$. The dashed curves show the total relative enstrophy in the system, again for the various values of $\hat{z}_{0} / \beta^{2}$. For $\beta \neq 1$ all the curves are multiplied by $\beta^{2}$. 


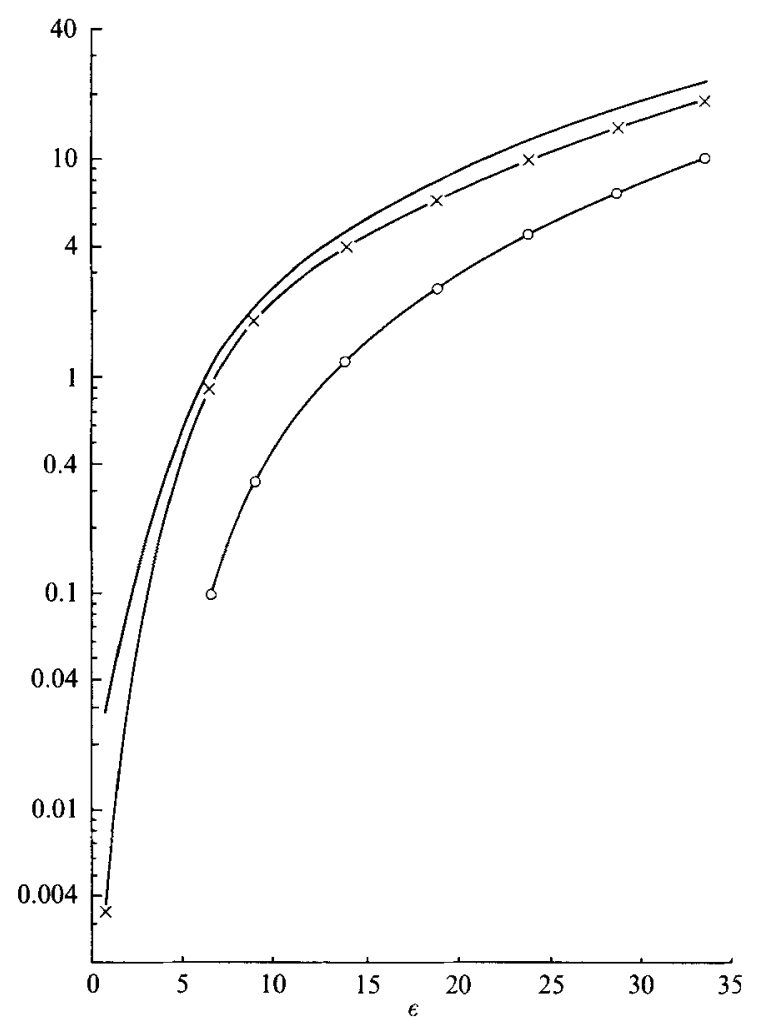

FIGURE 6. Comparison between the bound on the wavy enstrophy for the Bickley jet (upper curve) and the maximum wavy enstrophy obtained in numerical calculations by Kwon \& Mak (1988). The crosses denote results from inviscid calculations, and the open circles denote results from viscous calculations of the form (4.1)-(4.3) with $r=0.2$. In all cases $\beta=0.4$. For $\epsilon>1.2$ the best upper bound is given by (7.3), but for smaller $\epsilon$ it must be picked off from figure 5 .

and given $\epsilon$. The results are presented in figure 5, together with the bound (7.3); the latter is seen to be relatively feeble. Also plotted is the total amount of relative enstrophy in the system, which here is given by

$$
\hat{z}_{0}+2 u_{0}^{2} \int_{-\infty}^{\infty} \operatorname{sech}^{4} y \tanh ^{2} y \mathrm{~d} y=\hat{z}_{0}+\frac{8}{15} u_{0}^{2}
$$

The nonlinear equilibration of instabilities to the Bickley jet (in a bounded domain) has recently been studied numerically by Kwon \& Mak (1988). Although it is not possible to calculate the wavy enstrophy directly from their published results, they have kindly provided the dața shown in figure 6. For computational reasons Kwon \& Mak mainly focus on the case of large $\epsilon$, and in this regime the rigorous bound is seen to compare fairly closely with the inviscid calculations. The maximum amplitudes in a viscous run are, on the other hand, substantially smaller.

\subsection{The free shear layer}

Next consider the free shear layer

$$
\widetilde{U}_{0}(y)=u_{0} \tanh y, \quad \bar{P}_{0}(y)=\beta y-u_{0} \operatorname{sech}^{2} y \quad(-\infty<y<\infty),
$$


(a)

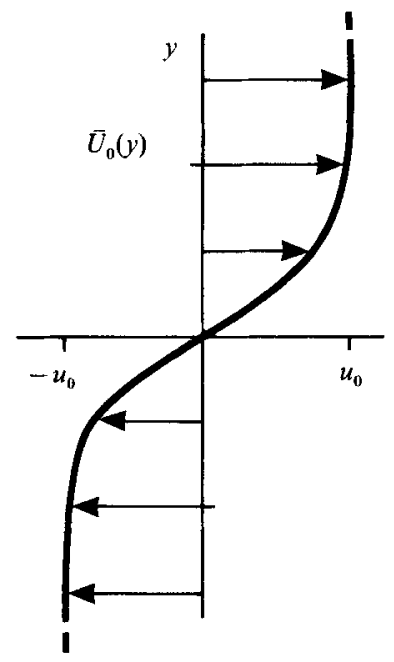

(b)

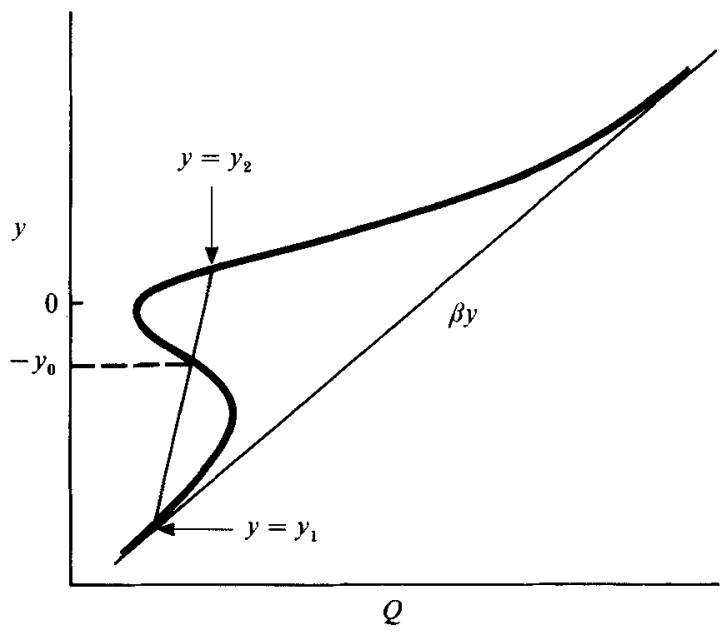

Figure 7. (a) The flow profile $\bar{U}_{0}(y)$ for the free shear layer (7.8). (b) The corresponding $\bar{P}_{0}(y)$ profile, together with the basic flow described in the text (thin line).

shown in figure 7 . This is known to be unstable for $u_{0}>u_{\text {crit }}=3^{\frac{3}{2}} \beta / 4$. Once again, one may appeal to the formulae of $\$ 5$, using

$$
C=4 \times 3^{-\frac{3}{2}}, \quad c=-4 \times 3^{-\frac{3}{2}}, \quad \gamma=-1, \quad Z=\frac{4}{3},
$$

and refer to figure $3(b)$. In the special case $z_{0} \rightarrow 0(5.16)$ gives

$$
\int_{-\infty}^{\infty} \frac{1}{2} \overline{\left(\nabla^{2} \bar{\Phi}^{\prime}\right)^{2}} \mathrm{~d} y \leqslant\left\{\begin{array}{ll}
\frac{9}{2} \beta^{2} \epsilon(2-\epsilon) & \text { for } \epsilon \leqslant 0.2 \\
\frac{9}{8} \beta^{2}(1+\epsilon)^{2} & \text { for } \epsilon \geqslant 0.2 .
\end{array}\right\}
$$

As with the previous example, the bound (7.10) may be improved upon significantly. The most negative value of $\mathrm{d} \bar{P}_{0} / \mathrm{d} y$ occurs at $y=-y_{0}$, where $y_{0} \equiv \operatorname{arctanh}(1 / \sqrt{ } 3) \approx 0.66$. The new family of stable basic states is constructed, similarly to that given by (7.4), by taking a straight line of (positive) slope $\alpha$ through the point $(y, Q)=\left(-y_{0}, \bar{P}_{0}\left(-y_{0}\right)\right)$; this will intersect $\bar{P}_{0}(y)$ again at the points $y=y_{1}$ and $y=y_{2}$, say. The construction is indicated in figure $7(b)$. For this family,

$$
\left|Q_{y}\right|_{\min }=\alpha, \quad\left|Q_{y}\right|_{\max }=\left\{\begin{array}{ll}
\bar{P}_{0}\left(y_{2}\right) & \text { if } y_{2} \geqslant y_{0} \\
\bar{P}_{0}\left(y_{0}\right)=\beta+4 \times 3^{-\frac{3}{2}} u_{0} & \text { if } y_{2} \leqslant y_{0},
\end{array}\right\}
$$

and

$$
\int_{-\infty}^{\infty} \frac{1}{2} \overline{q^{2}(0 ; \alpha)} \mathrm{d} y=\int_{-\infty}^{\infty} \frac{1}{2} \overline{\left(\nabla^{2} \Phi_{0}^{\prime}\right)^{2}} \mathrm{~d} y+\int_{y_{1}}^{y_{2}} \frac{1}{2}\left\{(\beta-\alpha)\left(y+y_{0}\right)+\frac{2}{3} u_{0}-u_{0} \operatorname{sech}^{2} y\right\}^{2} \mathrm{~d} y
$$

as before, the second term on the right-hand side of (7.12) may be integrated exactly in terms of $y_{1}$ and $y_{2}$, and $y_{1}$ and $y_{2}$ may be found numerically using an iterative root finder. The minimum over $\alpha$ of the right-hand side of (3.4), using (7.11) and (7.12), has been calculated numerically, and is compared with (7.10) in figure 8 . It is evident that this bound is significantly better than (7.10) over a very wide range of $\epsilon$. Also 


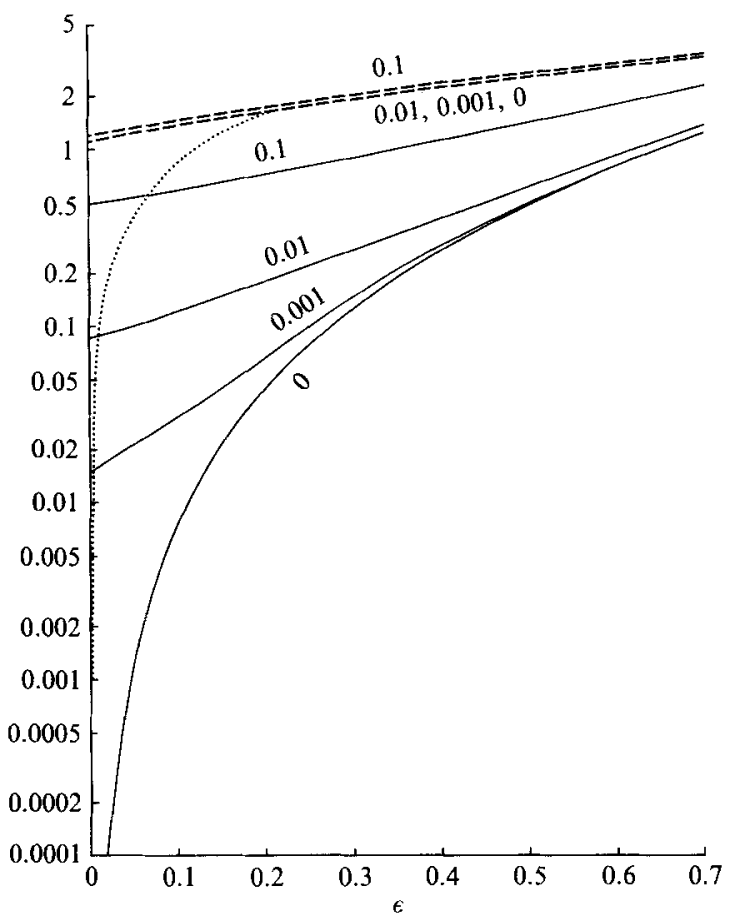

Figure 8. The bound on the wavy enstrophy for the free shear layer, as a function of the supereriticality $\epsilon$, for $\beta=1$. The dotted curve shows (7.10), relevant to the limit $z_{0} \rightarrow 0$. The solid curves indicate the minimum over $\alpha$ of (3.4), using (7.11) and (7.12), for various $\hat{z}_{0}$; the number denotes the value of $\hat{z}_{0} / \beta^{2}$. The dashed curves show the total relative enstrophy in the system, again for the various values of $\hat{z}_{0} / \beta^{2}$. For $\beta \neq 1$ all the curves are multiplied by $\beta^{2}$.

plotted is the total amount of relative enstrophy in the system, which here is given by

$$
\hat{z}_{0}+\frac{1}{2} u_{0}^{2} \int_{-\infty}^{\infty} \operatorname{sech}^{4} y \mathrm{~d} y=\hat{z}_{0}+\frac{2}{3} u_{0}^{2}
$$

Churilov \& Shukhman (1987) and Shukhman (1987) have studied the quasi-linear equilibration of instabilities to this profile by using weakly nonlinear perturbation expansions in the limit of small supercriticality. While the former reference considered only single-wave equilibration, the latter treated the more physically relevant case of an initial excitation consisting of a large number of waves with close phase velocities, and we shall therefore compare the saturation bounds derived above with Shukhman's (1987) theory. We are here especially interested in the inviscid case. (Shukhman's form of viscosity is vorticity diffusion, which is not of the form considered in $\$ 4$.) Taking the wavy perturbation to be a spectrum of waves whose spatial structure is given to leading order by the marginally stable mode at $k=k_{0}=\left(\frac{2}{3}\right)^{\frac{1}{2}}$ and $c=c_{0}=-1 / \sqrt{ } 3$, namely

$$
\Phi^{\prime}(x, y, t) \approx \sum_{k} A_{k}(t) \phi_{a}(y) \mathrm{e}^{\mathrm{i} k_{0}\left(x-c_{0} t\right)}, \quad \phi_{a}(y)=\mathrm{e}^{-c_{0} y} \operatorname{sech} y
$$

the wavy enstrophy is then given (to leading order) by

$$
\frac{1}{4} \sum_{k}\left|A_{k}\right|^{2} \int_{-\infty}^{\infty} \mathrm{e}^{-2 c_{0} y} \operatorname{sech}^{2} y\left\{\left(c_{0}^{2}-k_{0}^{2}-1\right)+2 \tanh ^{2} y+2 c_{0} \tanh y\right\}^{2} \mathrm{~d} y
$$




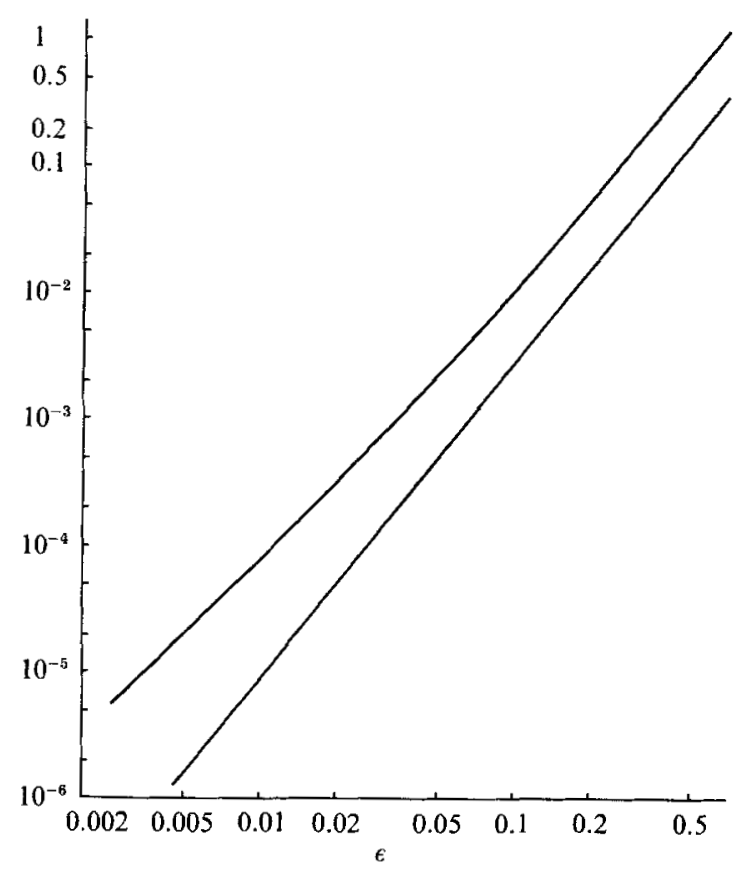

Figure 9. Comparison between the maximum enstrophy of the weakly nonlinear solution presented by Shukhman (lower curve), namely (7.17) with $\int w \mathrm{~d} z=4 \sqrt{ } 3 / 5$, and the rigorous upper bound corresponding to (7.11) and (7.12) for the relevant value of $\hat{z}_{0}$ (upper curve), the latter being (7.17) with $\int w \mathrm{~d} z=0.04$.

The integral in (7.14) has been solved exactly in terms of $c_{0}$ (see Appendix B), and for $c_{0}=-1 / \sqrt{ } 3$ takes a value of approximately 5.76. According to I. G. Shukhman (personal communication, 1987), and using his notation,

$$
\begin{aligned}
\sum_{k}\left|A_{k}\right|^{2} & =\frac{4\left|c_{0}\right|^{3}}{k_{0} \phi_{0}^{2}} \int W(\xi) \mathrm{d} \xi=\frac{4\left|c_{0}\right|^{3}}{k_{0} \phi_{0}^{2}} \frac{\gamma_{m}^{2} \mu \pi}{\left|c_{0}\right|}\left\{\frac{3 \Delta \beta}{8\left|c_{0}\right|}\right\}^{\frac{1}{2}} \int w(z) \mathrm{d} z \\
& \approx 0.44(\Delta \beta)^{\frac{5}{2}} \int w(z) \mathrm{d} z
\end{aligned}
$$

and note that in terms of the shear supercriticality $\epsilon \equiv\left(u_{0}-u_{\text {crit }}\right) / u_{\text {crit }}$,

$$
\Delta \beta \equiv \beta_{\text {erit }}-\beta=4 \times 3^{-\frac{3}{2}} u_{0}-\beta=\beta\left\{\frac{u_{0}}{u_{\text {erit }}}-1\right\}=\beta \epsilon .
$$

Then using the relation

$$
\int_{-\infty}^{\infty} \frac{1}{2} \overline{\left(\nabla^{2} \Phi^{\prime}\right)^{2}} \mathrm{~d} y=\frac{1}{4}(0.44)(5.76) \beta^{\frac{5}{2}} \epsilon^{\frac{5}{2}} \int w(z) \mathrm{d} z,
$$

it is possible to convert Shukhman's w's to wavy enstrophies. In the calculation presented in Shukhman's figure $3, \int w \mathrm{~d} z$ reaches a maximum of $4 \sqrt{ } 3 / 5$ when initialized at 0.04 (personal communication, 1987). The weakly nonlinear results, for equilibration of this multiwave spectrum, are compared in figure 9 with the rigorous upper bound derived above, using the relevant value of $\hat{z}_{0}$ (based on (7.17) with $\left.\int w \mathrm{~d} z=0.04\right)$. The agreement is seen to be reasonably close over a wide range of $\epsilon$. It is interesting to note that in Shukhman's calculation the equilibration occurs via a 

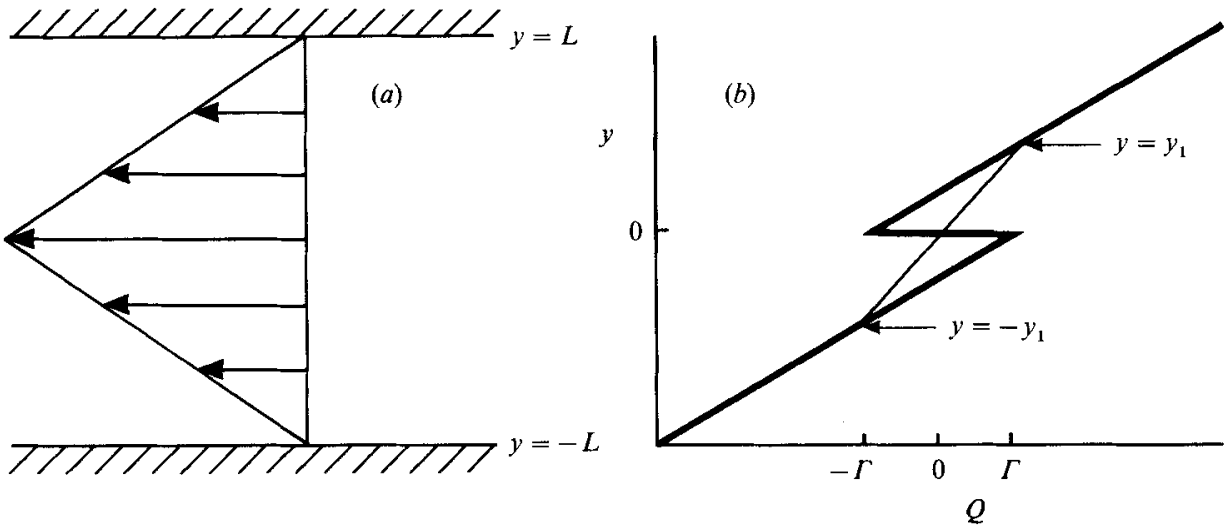

FiaURe 10. (a) The flow profile $\bar{U}_{0}(y)$ for the point jet (7.18), with $u_{0}=-\Gamma L$. (b) The corresponding $\bar{P}_{0}(y)$ profile, together with the basic flow (7.19) (thin line).

rearrangement of vorticity in the vicinity of the critical layer. For the most unstable mode the critical layer is at $y_{\mathrm{c}}=-\operatorname{arctanh}(1 / \sqrt{ } 3)$, which is also the point where $\mathrm{d} \bar{P}_{0} / \mathrm{d} y$ is most negative. The bound derived from (7.11) and (7.12) was obtained by picking a basic flow that differed from (7.8) in precisely that region.

\subsection{The point jet}

The next example is the so-called 'point jet', consisting of the broken-line profile

$$
\bar{U}_{0}(y)=u_{0}+\Gamma|y|, \quad \bar{P}_{0}(y)=\beta y-\operatorname{sgn}(y) \Gamma \quad(-L<y<L),
$$

with discontinuous $\bar{P}_{0}(y)$, shown in figure 10 . This flow is unstable for any value of $\beta$, and therefore to apply the Rayleigh theorem (2.5) one must choose a family of basic flows having quite different structure. The family

$$
Q(y ; \alpha)=\left\{\begin{array}{ll}
\beta y+\Gamma & \text { for } y<-y_{1} \\
\alpha y & \text { for }-y_{1}<y<y_{1} \\
\beta y-\Gamma & \text { for } y>y_{1}
\end{array}\right\}
$$

is plausible, being stable for $\alpha>0$; to ensure continuity of $Q$ it is necessary to take $y_{1}=\Gamma /(\beta-\alpha)$, and we presume $y_{1}<L$. A profile of the type (7.19) is shown in figure $10(b)$. For such a basic flow, we have

$$
\left|Q_{y}\right|_{\max }=\beta, \quad\left|Q_{y}\right|_{\min }=\alpha,
$$

and

$$
\begin{aligned}
\int_{-L}^{L} \frac{1}{2} \overline{q^{2}(0 ; \alpha)} \mathrm{d} y & =\int_{-L}^{L} \frac{1}{2} \overline{\left(\nabla^{2} \Phi_{0}^{\prime}\right)^{2}} \mathrm{~d} y+\int_{0}^{y_{1}}(\beta y-\Gamma-\alpha y)^{2} \mathrm{~d} y \\
& =\hat{z}_{0}+\frac{\Gamma^{3}}{3(\beta-\alpha)}
\end{aligned}
$$

where $\hat{z}_{0}$ is the initial wavy enstrophy (as before). Thus the right-hand side of (3.4) may be written as

$$
\frac{\beta}{\alpha}\left\{\hat{z}_{0}+\frac{\Gamma^{3}}{3(\beta-\alpha)}\right\}=\frac{\beta \Gamma^{3}}{3}\left\{\frac{z_{0}^{\prime}}{\alpha \beta}+\frac{1}{\alpha(\beta-\alpha)}\right\},
$$


where $z_{0}^{\prime} \equiv 3 \beta \hat{z}_{0} / \Gamma^{3}$, and is minimized for the choice

$$
\alpha=\alpha_{\min } \equiv \frac{\beta}{z_{0}^{\prime}}\left\{\left(1+z_{0}^{\prime}\right)-\left(1+z_{0}^{\prime}\right)^{\frac{1}{2}}\right\}
$$

In the limit of small $z_{0}^{\prime},(7.23)$ may be approximated as

$$
\alpha_{\min } \approx \frac{1}{2} \beta\left\{1+\frac{1}{4} z_{0}^{\prime}+O\left(z_{0}^{\prime 2}\right)\right\}
$$

substituting (7.24) into (7.22) then gives the asymptotic result

$$
\int_{-L}^{L} \frac{1}{2} \overline{\left(\nabla^{2} \Phi^{\prime}\right)^{2}} \mathrm{~d} y \leqslant \frac{\Gamma^{3}}{3 \beta}\left\{4+2 z_{0}^{\prime}+O\left(z_{0}^{\prime 2}\right)\right\}
$$

valid in the limit $z_{0}^{\prime} \rightarrow 0$. Note that the bound is independent of $L$.

Schoeberl \& Lindzen (1984) have recently performed a numerical study of the saturation of the point-jet instability; they argue that a useful estimate (though not an upper bound) for the wavy enstrophy is that required to make the zonal flow neutrally stable. This quantity is given by (7.21) with $\hat{z}_{0}=0$ and $\alpha=0$, and equals $\Gamma^{3} / 3 \beta$; this is one-fourth of the small- $z_{0}^{\prime}$ limit of (7.25). The numerical experiment presented in their figure 8 , with $z_{0}^{\prime} \ll 1$, shows the wavy enstrophy overshooting the 'saturation limit' $\Gamma^{3} / 3 \beta$ by a factor of two, coming within a factor of two of the rigorous upper bound (7.25).

\subsection{A strip of relative vorticity}

The final example of this section is a strip of relative vorticity, equivalently a layer of constant shear,

$$
\bar{U}_{0}(y)=\left\{\begin{array}{ll}
u_{0} & \text { for } y \geqslant d \\
\Gamma y & \text { for }-d \leqslant y \leqslant d \\
-u_{0} & \text { for } y \leqslant-d,
\end{array}\right\} \quad \bar{P}_{0}(y)=\left\{\begin{array}{ll}
\beta y & \text { for }|y|>d \\
\beta y-\Gamma & \text { for }|y|<d
\end{array}\right\}
$$

with $\Gamma \equiv u_{0} / d$. The zonal flow (7.26) is sketched in figure 11, and clearly bears a certain resemblance to the free shear layer. In the limit $d \rightarrow 0$ the strip becomes a vortex sheet. The flow (7.26) is unstable for any $\beta$, so we construct a family of basic states according to

$$
Q(y ; \alpha)=\left\{\begin{array}{ll}
\beta y & \text { for }|y| \geqslant d+\alpha \\
\beta y-\frac{\Gamma}{\alpha}(\alpha+d-|y|) & \text { for } d \leqslant|y| \leqslant d+\alpha \\
\beta y-\Gamma & \text { for }|y| \leqslant d
\end{array}\right\}
$$

with $\alpha>\Gamma / \beta$, shown in figure $11(b)$. For such a basic state we have

$$
\left|Q_{y}\right|_{\max }=\beta+\frac{\Gamma}{\alpha}, \quad\left|Q_{y}\right|_{\min }=\beta-\frac{\Gamma}{\alpha},
$$

and

$$
\int_{-\infty}^{\infty} \frac{1}{2} \overline{q^{2}(0 ; \alpha)} \mathrm{d} y=\int_{-\infty}^{\infty} \frac{1}{2} \overline{\left(\nabla^{2} \Phi_{0}^{\prime}\right)^{2}} \mathrm{~d} y+\int_{0}^{\alpha}\left\{\frac{\Gamma x}{\alpha}\right\}^{2} \mathrm{~d} x=\hat{z}_{0}+\frac{1}{3} \Gamma^{2} \alpha .
$$

The right-hand side of (3.4) may therefore be written as

$$
\frac{\alpha \beta+\Gamma}{\alpha \beta-\Gamma}\left\{\hat{z}_{0}+\frac{1}{3} \Gamma^{2} \alpha\right\}=\frac{\alpha \beta+\Gamma}{\alpha \beta-\Gamma}\left\{z_{0}^{\prime}+\frac{\beta \alpha}{\Gamma}\right\} \frac{\Gamma^{3}}{3 \beta},
$$



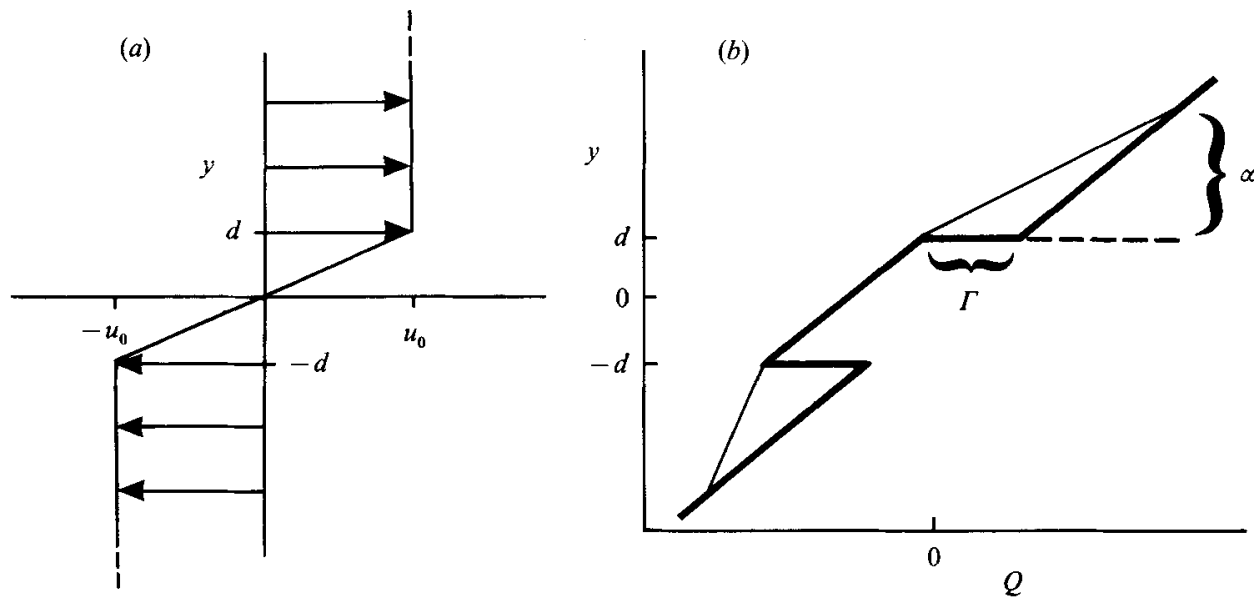

Figure 11. (a) The flow profile $\bar{U}_{0}(y)$ for the vortex strip (7.26). (b) The corresponding $\bar{P}_{0}(y)$ profile, together with the basic flow (7.27) (thin line).

where $z_{0}^{\prime} \equiv 3 \beta \hat{z}_{0} / \Gamma^{3}$, and is minimized for the choice

$$
\alpha=\alpha_{\min } \equiv \frac{\Gamma}{\beta}\left\{1+\left(2\left(1+z_{0}^{\prime}\right)\right)^{\frac{1}{2}}\right\} .
$$

In the limit of small $z_{0}^{\prime},(7.31)$ may be approximated as

$$
\alpha_{\min } \approx \frac{\Gamma}{\beta}\left\{1+\sqrt{ } 2\left(1+\frac{1}{2} z_{0}^{\prime}\right)+O\left(z_{0}^{\prime 2}\right)\right\}
$$

substituting (7.32) into (7.30) then gives the asymptotic result

$$
\int_{-\infty}^{\infty} \frac{1}{2} \overline{\left(\nabla^{2} \Phi^{\prime}\right)^{2}} \mathrm{~d} y \leqslant \frac{\Gamma^{3}}{3 \beta}\left\{3+2 \sqrt{ } 2+(\sqrt{ } 2+1) z_{0}^{\prime}+O\left(z_{0}^{2}\right)\right\}
$$

valid in the limit $z_{0}^{\prime} \rightarrow 0$. It is interesting to compare this bound with the total amount of relative enstrophy in the strip, which is $d \Gamma^{2}$. Thus a total breakup of the strip can be definitely precluded (in the limit $z_{0}^{\prime} \rightarrow 0$ ) whenever

$$
\frac{\Gamma^{3}}{3 \beta}(3+2 \sqrt{ } 2)<d \Gamma^{2} \Leftrightarrow \frac{u_{0}}{\beta d^{2}}<\frac{3}{3+2 \sqrt{ } 2} .
$$

As expected, breakup can be prevented neither in the vortex-sheet limit $d \rightarrow 0$, nor in the limit $\beta \rightarrow 0$. The flow in both these limits is of course known to be strongly unstable.

\section{Discussion}

In this paper, a new algorithm has been presented for obtaining rigorous bounds on the nonlinear saturation of instabilities to parallel shear flows on the beta-plane. The method relies on the existence of finite-amplitude Liapunov stability theorems $(\S 2)$, which bound the evolution of disturbance norms about stable basic flows. It may seem odd that a stability theorem can constrain the behaviour of an unstable flow, but such is indeed the case. The point is that if the unstable flow is close enough 
to a stable flow - in a sense that can be made mathematically precise - then the former can be regarded as a finite-amplitude disturbance to the latter, and can be usefully constrained by the nonlinear stability theorem. The present approach can be regarded as complementary to the linearized study of Pierrehumbert (1983), which showed that steady states that are close to a stable inviscid steady state are at worst weakly unstable, in the sense that disturbance growth rates are small.

Various applications of these bounds suggest themselves. First, since the bounds are rigorous, they provide one way of delineating the range of validity of weakly nonlinear theories. Second, whenever the bounds are useful, in the sense that they prohibit a total breakup of the parallel flow into non-parallel motion (to the extent permitted by impulse conservation), the flow can be considered non-ergodic with respect to the phase-space hypersurface of constant energy, enstrophy, and impulse (cf. Shepherd 1987). This latter result has implications concerning certain statistical assumptions commonly used in geostrophic turbulence theory (see e.g. Salmon 1982). Third, the bounds give parameter dependences for finite-amplitude saturation which (unlike those from weakly nonlinear theories) are unconstrained by any assumptions about the nature of the initial wavy disturbance. Whether the maximum amplitude of the non-parallel part of the flow is accurately predicted, in any given realization, by the bounds derived above is a matter for numerical experimentation. But the fairly close agreement with weakly nonlinear and numerical solutions, seen in the examples of $\$ \S 7.1-7.3$, is certainly encouraging. Similarly encouraging results are found elsewhere (Shepherd 1988) for the baroclinic-instability problem.

A weakness of the approach is that there is no way of determining a priori whether the best bounds obtained from the consideration of a certain family of stable basic flows could not be improved upon by considering a somewhat wider family. The examples of $\S 7.1$ and $\$ 7.2$ show that different families of stable basic flows can yield bounds which are quantitatively quite different (although the parameter dependences turn out to be similar). In the present study, attention has been restricted to rather simple kinds of families. To allow a much more complicated range of stable basic flows would lead one to a non-trivial problem of multi-parameter optimization, but such an approach is certainly feasible for any particular application. However, in this regard it is important to note the following point (due to Dr P. H. Haynes): any particular saturation bound may be improved upon (though possibly only slightly) if the basic-state absolute-vorticity gradient $Q_{y}$ varies smoothly in the neighbourhood of a point where it takes an extremum. This means that one is justified in focusing attention on functions $Q_{y}$ that take their extrema over finite intervals, as the best bounds will be found by using such functions (cf. $\$ 7$ ). The detailed argument is given in Appendix $\mathrm{C}$.

One interesting insight from the present work is a resolution of an apparent dilemma posed by Andrews' (1984) theorem. Andrews presented an argument which implied that if the geometry of a problem is zonally symmetric (i.e. $x$-invariant), then any flow that is provably stable by Arnol'd-type theorems (namely the generalized Rayleigh and Fjørtoft theorems discussed in $\$ 2$ ) must itself be zonally symmetric. This implies that even slightly non-zonal flows are not provably stable, perhaps leading one to question the physical relevance of the Arnol'd theorems. (There is in fact a flaw in Andrews' reasoning in the case of an unbounded fluid, possibly connected with the fact that the variations that he uses to derive his result are not generally admissible. A simple counter-example to the theorem is that circular vortices in an infinite domain may be provably stable by the generalized Rayleigh 
theorem. Moreover, Andrews' argument could equally well use $y$ in place of $x$, and would then lead to the absurd conclusion that even zonal flows could not be provably stable. However, Andrews' result does apply to a zonal channel geometry.) In any case the dilemma is easily resolved by the present work, which suggests that a slightly non-zonal flow may be regarded as a small disturbance to a zonal flow; if the latter is stable, then any instability to the non-zonal flow must saturate at an amplitude proportional to the degree of non-zonality. Therefore, provided the underlying zonal flow is stable, the saturation of disturbances to non-zonal flows is continuous in the limit of small non-zonality.

One is perhaps led to wonder about the possible connection between the present approach and the energy-stability method (e.g. Howard 1972; Joseph 1976, §4) for determining upper bounds on quadratic flow quantities. However, the energystability method relies explicitly on non-zero viscosity (with bounds that diverge in the limit of infinite Reynolds number); it is restricted to flows in confined geometries; and it takes no apparent account of dynamical stability mechanisms. Therefore any resemblance between the two approaches would appear to be superficial.

Finally, it may be mentioned that the approach described in this paper is clearly not restricted to parallel flows. When the geometry of the problem is not zonally symmetric (for example because of topography) then there will generally be nonparallel states which are provably stable by one of Arnol'd's (1966) stability theorems (see MeIntyre \& Shepherd 1987, §6), and instabilities to non-parallel basic flows may possibly be bounded if these stable flows are sufficiently close. In this case the bound would be on a combination of the energy and enstrophy, as with the generalized Fjørtoft theorem (2.6).

The author would like to thank M. Mak and H. J. Kwon for providing the data in figure 6, I. G. Shukhman for helping to compare his results with those derived here, and P. H. Haynes for many useful comments (including Appendix C). This research has been supported by the UK Natural Environment Research Council and by St Catharine's College, Cambridge.

\section{Appendix A. Nonlinear stability theorems for piecewise-constant distributions of vorticity}

For some fluid-dynamical applications, a useful simplifying idealization is provided by considering flows with piecewise-constant distributions of vorticity. Taken at face value, the finite-amplitude stability theorems (2.5) and (2.6) would appear to be invalid for such problems because $Q_{y}$ is then composed of delta-functions. However, although the linearized versions of the pseudomomentum and pseudoenergy conservation laws do indeed break down in such circumstances, their finite-amplitude forms involve integrals rather than derivatives (see (4.4) and (4.15)) and therefore remain perfectly well defined. In this section, finite-amplitude stability theorems corresponding to (2.5) and (2.6) are derived for such problems.

\section{A.1. The generalized Rayleigh theorem}

We begin by considering the form of the pseudomomentum density (4.4) for the case of a disturbance to a basic flow

$$
Q(y)=\left\{\begin{array}{lll}
Q_{2} & \text { for } & y>0 \\
Q_{1} & \text { for } & y<0
\end{array}\right\}
$$


(a)

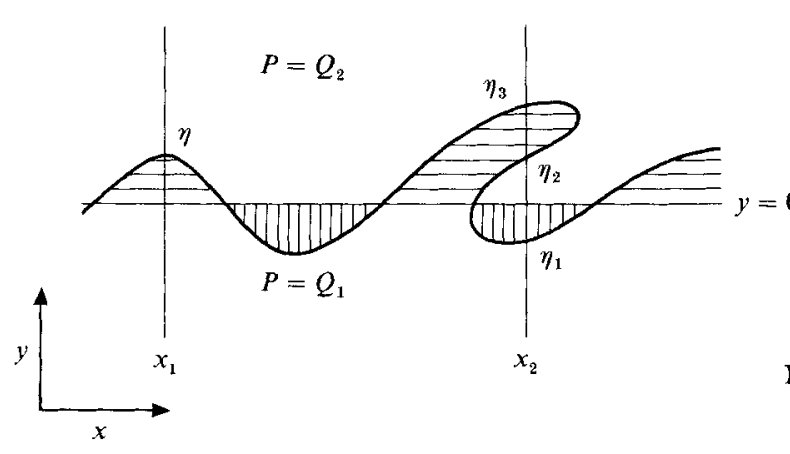

(b)

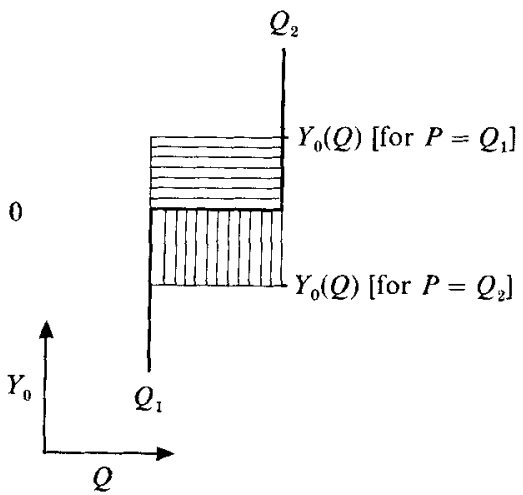

Figure 12. (a) Sketch of a hypothetical disturbance to the basic flow (A 1). The disturbance is defined by the position of the contour marking the vorticity jump. (b) The function $Y_{0}(Q)($ thick line). See text for details.

with $Q_{2}>Q_{1}$ for definiteness, as sketched in figure $12(a)$. The corresponding function $Y_{0}(Q)$ is shown in figure $12(b)$. It may be seen that the function $A(Q, q)$ defined by (4.4) is non-zero only when $P=Q+q$ differs from $Q$, so that the integration in $\tilde{q}$ is across the jump in $Q$; physically, this situation corresponds to the hatched regions in figure $12(a)$. For example, in the case $P=Q_{2}, Q=Q_{1}$ indicated by the vertically hatched regions in figure $12(a)$, the value of $A(Q, q)$ is simply minus the area of the vertically hatched region in figure $12(b)$, with $Y_{0}(Q)=y<0$, namely $\left(Q_{2}-Q_{1}\right) y$. (This is a straightforward application of the rules discussed by McIntyre \& Shepherd $(1987, \S 5)$ for the case of the pseudoenergy.) Similarly, in the horizontally hatched regions in figure $12(a)$, with $P=Q_{1}$ and $Q=Q_{2}$, the local density $A(Q, q)$ is just minus the area of the horizontally hatched region in figure $12(b)$, with $Y_{0}(Q)=y>0$, namely $-\left(Q_{2}-Q_{1}\right) y$.

Although $A(Q, q)$ is then apparently first order in the disturbance, one may easily obtain a second-order quantity by integration in $y$. For example, at $x=x_{1}$ one has

$$
\int_{-\infty}^{\infty} A(Q, q) \mathrm{d} y=\int_{0}^{\eta} A(Q, q) \mathrm{d} y=-\left(Q_{2}-Q_{1}\right) \int_{0}^{\eta} y \mathrm{~d} y=-\frac{1}{2}\left(Q_{2}-Q_{1}\right) \eta^{2}
$$

while at $x=x_{2}$,

$$
\begin{aligned}
\int_{-\infty}^{\infty} A(Q, q) \mathrm{d} y & =\int_{\eta_{1}}^{0} A(Q, q) \mathrm{d} y+\int_{\eta_{2}}^{\eta_{\mathrm{3}}} A(Q, q) \mathrm{d} y=\left(Q_{2}-Q_{1}\right)\left\{\int_{\eta_{1}}^{0} y \mathrm{~d} y-\int_{\eta_{2}}^{\eta_{3}} y \mathrm{~d} y\right\} \\
& =-\frac{1}{2}\left(Q_{2}-Q_{1}\right)\left(\eta_{3}^{2}-\eta_{2}^{2}+\eta_{1}^{2}\right) .
\end{aligned}
$$

The general expression for the global integral of $A(Q, q)$ may be written in the form

$$
\int \overline{A(Q, q)} \mathrm{d} y=-\frac{1}{2}\left(Q_{2}-Q_{1}\right) \overline{\oint \eta^{2} \mathrm{~d} x}
$$

with $\eta$ the contour displacement, the integral on the right-hand side being taken along the contour. In the case of multiple contours $C_{i}$, one simply sums the contribution from each contour according to

$$
\int \overline{A(\bar{Q}, q)} \mathrm{d} y=-\frac{1}{2} \sum_{i} \Delta Q_{i} \oint_{C_{i}} \eta^{2} \mathrm{~d} x .
$$


Now, the integrated pseudomomentum (A 3) is a constant of the motion, and stability will be implied whenever it is of definite sign for arbitrary disturbances. It is obvious from (A 3) that the latter condition will be true whenever the vorticity jumps $\Delta Q_{i}$ are of the same sign; this is clearly the condition that is analogous to monotonicity of $Q(y)$ in the continuous case. Unlike the continuous case, however, where the integrated pseudomomentum is generally not itself a norm (but can nevertheless be sandwiched between norms), here it does provide a norm, namely

$$
\|\eta\|^{2} \equiv\left|\int \overline{A(Q, q)} \mathrm{d} y\right|=\frac{1}{2} \sum_{i}\left|\Delta Q_{i}\right| \overline{\oint_{C_{i}} \eta^{2} \mathrm{~d} x} .
$$

Thus, when $\Delta Q_{i}$ are all of the same sign, Liapunov stability is assured in the sense that

$$
\|\eta(t)\|=\|\eta(0)\|
$$

These results have recently been derived by Dritschel (1988) by an alternative method. Contrary to the claims of Dritschel, it is evident that his results are in fact nothing more than a straightforward application of Arnol'd's method to the case of piecewise-constant vorticity.

\section{A.2. The generalized Fjortoft theorem}

We now consider the form of the pseudoenergy density (4.15) for the case of a disturbance to a basic flow

$$
Q(y)=\left\{\begin{array}{ll}
-\Gamma & \text { for } y<-d \\
\Lambda-\Gamma & \text { for }-d<y<d \\
-\Gamma & \text { for } y>d,
\end{array}\right\} \quad U(y)=\left\{\begin{array}{ll}
\Gamma y+\Lambda d & \text { for } y<-d \\
(\Gamma-\Lambda) y & \text { for }-d<y<d \\
\Gamma y-\Lambda d, & \text { for } y>d,
\end{array}\right\}
$$

with $A>0$ for definiteness, as sketched in figure 13. This may be considered a strip of positive vorticity $A$ in the presence of a shear flow $U \propto \Gamma y$. Because the vorticity jumps $\Delta Q$ are of opposite sign, the generalized Rayleigh theorem (A 5) does not apply; however, it will be shown below that the flow is nonlinearly stable whenever $(\Gamma-A) A>0$ in some frame of reference, which is precisely analogous to the Fjørtoft condition $U / Q_{y}<0$ (or, strictly speaking, to the equivalent condition $U Q_{y}<0$ ) in the continuous case. Thus we take $\Gamma>A$, which correponds to the case of 'adverse' shear $\Gamma$, as indicated in figure $13(b)$.

In the expression for the pseudoenergy density (4.15), the disturbance energy $E(Q, q)$ is positive definite; thus stability is provable whenever $B(Q, q)$ is also positive definite (this is Arnol'd's first stability theorem). The $\Psi(Q)$-function for the basic flow (A 6) is shown in figure $13(c)$; note that because of the antisymmetry of $U(y), \Psi(Q)$ folds back onto itself perfectly. As with $A(Q, q)$ in the previous subsection, here $B(Q, q)$ will be non-zero only when $P=Q+q$ differs from $Q$, so that the integration in $\tilde{q}$ is across the jump in $Q$. Consider first the contribution of $B(Q, q)$ due to undulations of the upper contour (originally at $y=d$ ), for which $\Psi_{y}<0$. In regions with $P=-\Gamma$ and $Q=A-\Gamma$, analogous to the vertically hatched regions in figure $12(a)$, the value of $B(Q, q)$ is simply the area of the vertically hatched region in figure $13(c)$, namely

$$
\Delta Q \Delta \Psi=-\Lambda\left\{\frac{1}{2}(\Gamma-\Lambda)\left(y^{2}-d^{2}\right)\right\},
$$

which is positive when $\Gamma>A$, since $y<d$ necessarily. $\dagger$ Similarly, in regions with $P=A-\Gamma$ and $Q=-\Gamma$, analogous to the horizontally hatched regions in figure $12(a)$,

$\dagger$ In fact, (A $7 a$ ) could be negative for an extremely large contour displacement with $y<-d$, but in that case the lower contour would give an even larger positive contribution. 
(a)

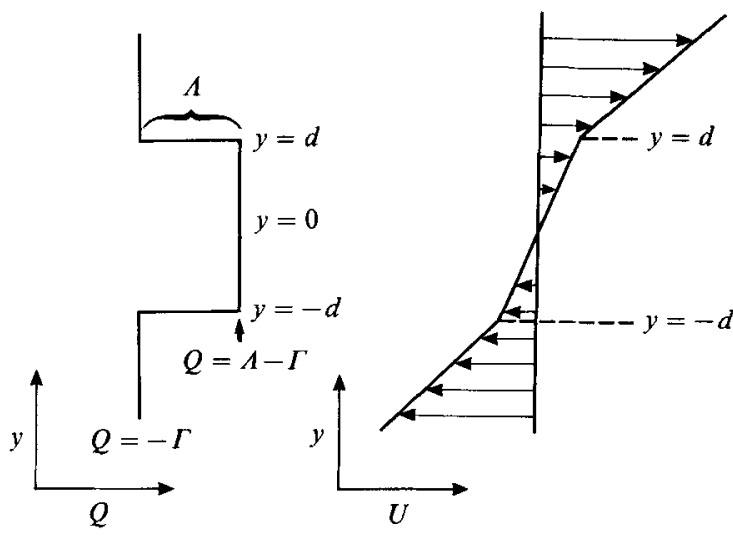

(c)

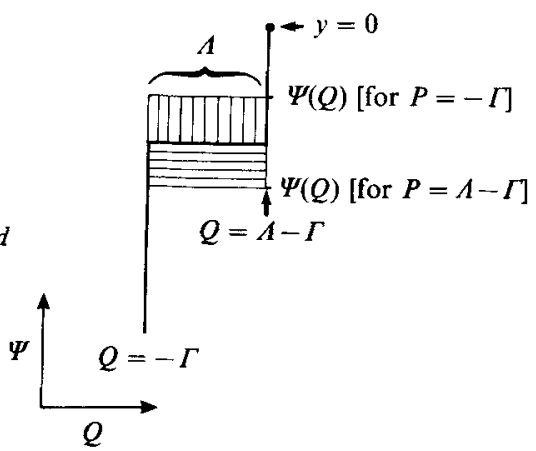

FIGURE 13. (a) The vorticity profile $Q(y),(b)$ the velocity profile $U(y)$, and $(c)$ the function $\Psi(Q)$ (thick line) for the basic flow (A 6).

the value of $B(Q, q)$ is the area of the horizontally-hatched region in figure $13(c)$, namely

$$
\Delta Q \Delta \Psi=\Lambda\left\{\frac{1}{2} \Gamma\left(y^{2}-d^{2}\right)-\Lambda d(y-d)\right\}
$$

which is also positive, since $y>d$ necessarily and $\Gamma>A$. It is obvious from symmetry considerations (and may be verified directly) that the contributions due to undulations of the lower contour - for which $\Psi_{y}>0$-are exactly the same, though with $(y-d)$ replaced by $-(y+d)$ in $(\mathrm{A} 7 b)$, which in this case applies to regions with $y<-d$.

Note that if one chose a frame of reference in which $U>0$ throughout the region of the vortex strip, then $\Psi_{y}<0$ for both the upper and lower contours, and the contribution to $B(Q, q)$ from undulations of the lower contour would be negative rather than positive. This situation is depicted in figure 14. Mathematically, the situation is entirely analogous to the continuous case; one seeks a frame of reference for which variations in $\Psi$ are always of the same sign as variations in $Q$, so that the pseudoenergy is positive definite, and this is true in figure 13 but not in figure 14 .

It is therefore evident that $\Gamma>A$ is a sufficient condition for the positive definiteness of the pseudoenergy. Since the latter is a constant of the motion, this establishes nonlinear stability in the first sense discussed by McIntyre \& Shepherd $(1987, \S 6)$; in particular, it rules out any temporally growing disturbance representable as a finite sum of modes having a prescribed spatial form (as is often assumed in linear and weakly nonlinear theories). Unfortunately, however, it is not at all clear how to derive a normed stability theorem analogous to (2.6); this would require introducing a disturbance norm which could be used to bound the pseudoenergy from both above and below. The technical obstacles appear to be twofold. First, both $(y-d)$ and $(y-d)^{2}$ appear in (A $\left.7 b\right)$, and one will dominate at small amplitude while the other will dominate at large amplitude. Second, $E(Q, q)$ depends on the disturbance stream function $\psi$ while $B(Q, q)$ depends on the contour displacement $\eta$, and in order to obtain the homogeneity property required of a norm one would have to establish similar dependences of $E$ and $B$ on the disturbance amplitude - something which is far from self-evident. 
(a)

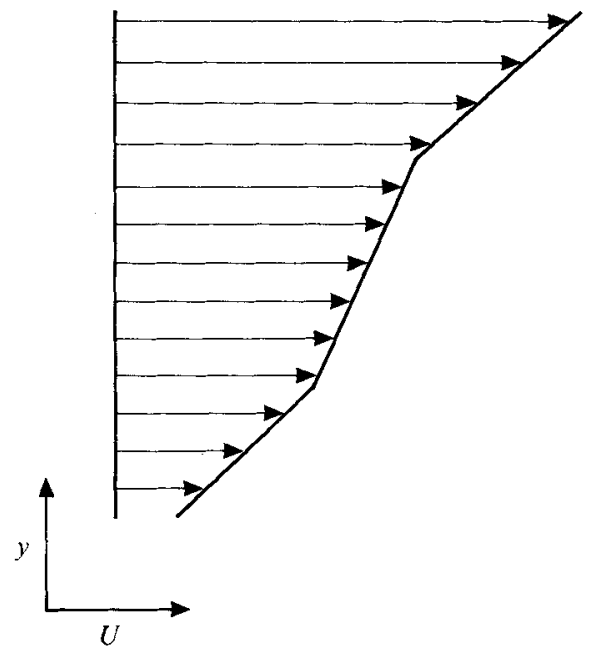

(b)

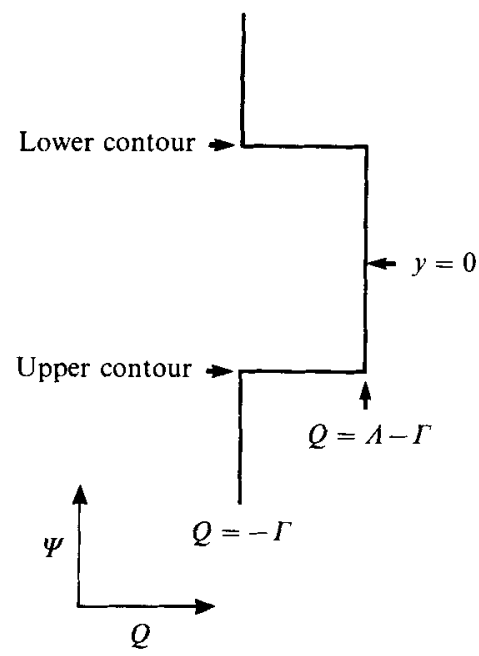

Figure 14. $(a)$ The velocity profile $U(y)$ and $(b)$ the function $\Psi(Q)$ for the basic flow (A 6 ), but in a shifted frame of reference for which the pseudoenergy is no longer of definite sign.

\section{Appendix B. Evaluation of the integral in (7.14)}

Making the substitution $x=\mathrm{e}^{y}$, the integral becomes

$$
\begin{aligned}
& \int_{0}^{\infty} x^{-2 c_{0}} \frac{4 x}{\left(x^{2}+1\right)^{2}}\left\{\left(c_{0}^{2}-k_{0}^{2}-1\right)+2 \frac{\left(x^{2}-1\right)^{2}}{\left(x^{2}+1\right)^{2}}+2 c_{0} \frac{\left(x^{2}-1\right)}{\left(x^{2}+1\right)}\right\}^{2} \mathrm{~d} x \\
& =4\left(c_{0}^{2}-k_{0}^{2}-1\right)^{2} \int_{0}^{\infty} \frac{x^{-2 c_{0}+1}}{\left(x^{2}+1\right)^{2}} \mathrm{~d} x+16 c_{0}\left(c_{0}^{2}-k_{0}^{2}-1\right) \int_{0}^{\infty} \frac{x^{-2 c_{0}+3}-x^{-2 c_{0}+1}}{\left(x^{2}+1\right)^{3}} \mathrm{~d} x \\
& \quad+16\left(2 c_{0}^{2}-k_{0}^{2}-1\right) \int_{0}^{\infty} \frac{x^{-2 c_{0}+5}-2 x^{-2 c_{0}+3}+x^{-2 c_{0}+1}}{\left(x^{2}+1\right)^{4}} \mathrm{~d} x \\
& \quad+32 c_{0} \int_{0}^{\infty} \frac{x^{-2 c_{0}+7}-3 x^{-2 c_{0}+5}-3 x^{-2 c_{0}+3}+x^{-2 c_{0}+1}}{\left(x^{2}+1\right)^{5}} \mathrm{~d} x \\
& \quad+16 \int_{0}^{\infty} \frac{x^{-2 c_{0}+9}-4 x^{-2 c_{0}+7}+6 x^{-2 c_{0}+5}-4 x^{-2 c_{0}+3}+x^{-2 c_{0}+1}}{\left(x^{2}+1\right)^{6}} \mathrm{~d} x .
\end{aligned}
$$

Each of these separate integrals may be written in terms of gamma-functions, using the relation

$$
\int_{0}^{\infty} \frac{x^{\mu-1}}{\left(x^{2}+1\right)^{n+1}}=\frac{\Gamma\left(\frac{1}{2} \mu\right) \Gamma\left(1+n-\frac{1}{2} \mu\right)}{2 \Gamma(n+1)}
$$

(Gradshteyn \& Ryzhik 1980, equation (3.241.4)), which yields factors of the form $\Gamma\left(n+c_{0}\right) \Gamma\left(m-c_{0}\right)$ for $1 \leqslant n, m \leqslant 5$. Then by using the recursion relation

$$
\Gamma(x+1)=x \Gamma(x)
$$

these factors may be expressed in terms of a polynomial in $c_{0}$ times $\Gamma\left(c_{0}\right) \Gamma\left(1-c_{0}\right)$, e.g.

$$
\Gamma\left(3-c_{0}\right) \Gamma\left(2+c_{0}\right)=\left(2-c_{0}\right)\left(1-c_{0}\right)\left(1+c_{0}\right) c_{0} \Gamma\left(c_{0}\right) \Gamma\left(1-c_{0}\right),
$$


and finally one may appeal to the relation

$$
\Gamma\left(c_{0}\right) \Gamma\left(1-c_{0}\right)=\pi \operatorname{cosec}\left(\pi c_{0}\right) .
$$

A rather complicated (but exact) expression in terms of $c_{0}$ results; evaluating it for $c_{0}=-1 / \sqrt{ } 3$ yields a value of approximately 5.76 .

\section{Appendix C. Demonstration that any smooth extremum of $Q_{y}$ allows an improvement to the saturation bound}

By P. H. Haynes

Department of Applied Mathematics and Theoretical Physics, University of Cambridge, Silver Street, Cambridge CB3 $9 E W, U K$

For a given initial (unstable) zonal flow $\left(\bar{\Phi}_{0}, \bar{P}_{0}\right)$ and initial wavy enstrophy $\int \frac{1}{2}\left(\overline{\left.\nabla^{2} \Phi_{0}^{\prime}\right)^{2}} \mathrm{~d} y\right.$, the object is to minimize the right-hand side of $(3.4)$,

$$
\frac{\left|Q_{y}\right|_{\max }}{\left|Q_{y}\right|_{\min }}\left\{\int \frac{1}{2} \overline{\left(\nabla^{2} \Phi_{0}^{\prime}\right)^{2}} \mathrm{~d} y+\int \frac{1}{2}\left(\bar{P}_{0}(y)-Q(y)\right)^{2} \mathrm{~d} y\right\} \equiv G\left[\bar{P}_{0}, Q\right]
$$

which represents a rigorous upper bound to the wavy enstrophy at any time $t$. The problem of finding a basic flow $Q(y)$ such that $\delta G=0$ may be addressed using the calculus of variations, but some insight into the nature of the solution may be obtained by considering the change in $G$ associated with a small change in the basic flow $Q$. In particular, we take $Q_{y}>0$ for definiteness and suppose that $Q_{y}$ takes a maximum at a single point $y_{0}$, and that $Q_{y}$ is continuously differentiable in a neighbourhood of $y_{0}$. We now demonstrate by explicit construction that the saturation bound $G$ associated with such a $Q$ cannot be a minimum.

In general, $Q_{y}$ can be represented near $y_{0}$ as

$$
Q_{y}(y)=Q_{y \max }-c\left(y-y_{0}\right)^{2}+O\left(\left(y-y_{0}\right)^{3}\right)
$$

with $c>0$. We now consider the variation in $G$ associated with a particular variation $\delta Q$ to $Q$. We choose $\delta Q$ to be a continuously differentiable function such that

(i) $\delta Q=0$ for $y \notin\left[y_{0}-\Delta, y_{0}+\Delta\right]$ and

(ii) $\delta Q_{y}<c\left(y-y_{0}\right)^{2}$ for $y \in\left[y_{0}-\Delta, y_{0}+\Delta\right]$

for some $\Delta$. For sufficiently small $\Delta$, condition (ii) ensures that $Q_{y}+\delta Q_{y}<Q_{y \max }$ in the interval $\left[y_{0}-\Delta, y_{0}+\Delta\right]$. A suitable choice for $\delta Q$ is the function

$$
\begin{gathered}
\delta Q=-\frac{1}{2} c \epsilon\left(y-y_{0}\right) \exp \left\{-\left(y-y_{0}\right)^{2} / 2 \epsilon\right\}+O\left(\epsilon \Delta \mathrm{e}^{-\Delta^{2} / 2 \epsilon}\right), \\
\delta Q_{y}=-\frac{1}{2} c \epsilon\left(1-\epsilon^{-1}\left(y-y_{0}\right)^{2}\right) \exp \left\{-\left(y-y_{0}\right)^{2} / 2 \epsilon\right\}+O\left(\Delta^{2} \mathrm{e}^{-\Delta^{2} / 2 \epsilon}\right),
\end{gathered}
$$

in the interval $\left[y_{0}-\Delta, y_{0}+\Delta\right]$, and $\delta Q \equiv 0$ outside, with $\epsilon$ a small parameter. In particular, let $\epsilon \ll \Delta^{2}$. Then

while

$$
\max \left\{Q_{y}+\delta Q_{y}\right\}=Q_{y \max }-\frac{1}{2} c \epsilon+O\left(\epsilon^{2}\right)+O\left(\Delta^{2} \mathrm{e}^{-\Delta^{2} / 2 \epsilon}\right),
$$

$$
\begin{aligned}
\int \frac{1}{2}\left(\bar{P}_{0}-Q-\delta Q\right)^{2} \mathrm{~d} y= & \int \frac{1}{2}\left(\bar{P}_{0}-Q\right)^{2} \mathrm{~d} y+\frac{1}{2} \epsilon \int\left(\bar{P}_{0}-Q\right) c\left(y-y_{0}\right) \\
& \exp \left\{-\left(y-y_{0}\right)^{2} / 2 \epsilon\right\} \mathrm{d} y+O\left(\epsilon^{2}\right) \\
= & \int \frac{1}{2}\left(\bar{P}_{0}-Q\right)^{2} \mathrm{~d} y+O\left(\epsilon^{2}\right) .
\end{aligned}
$$


Equation (C 4) shows that, under the variation $\delta Q,\left|Q_{y}\right|_{\max }$ decreases by $O(\epsilon)$, while (C 5) implies that the integral quantity in (C 1) increases by no more than $O\left(\epsilon^{2}\right)$. Together these demonstrate that

$$
\delta G<0
$$

for sufficiently small $\epsilon$, and $G\left[\bar{P}_{0}, Q\right]$ therefore cannot be a minimum.

A simple extension of this method deals with cases where $c=0$ (and some higher and even derivative of $Q_{y}$ is non-zero), and with the behaviour near the minimum value of $Q_{y}$. We deduce that $G$ cannot be minimized by a choice of $Q$ for which $Q_{y}$ is varying smoothly in a neighbourhood about the point where it attains its minimum or maximum value. In addition we see that $G$ is decreased if $Q$ is changed in such a way as to flatten out $Q_{y}$ near its maximum or minimum points. This is a strong indication that the minimum value of $G$ is attained by a $Q$ that has $Q_{y}$ taking its maximum and minimum values over finite intervals.

\section{REFERENCES}

ANDREws, D. G. 1984 On the existence of nonzonal flows satisfying sufficient conditions for stability. Geophys. Astrophys. Fluid Dyn. 28, 243-256.

ARnoL'D, V. I. 1966 On an a priori estimate in the theory of hydrodynamical stability. Izv. Vyssh. Uchebn.Zaved. Matematika 54, no. 5, 3-5. (English transl. : Amer. Math. Soc. Transl., Series 279, $267-269(1969)$.)

Bronshtein, I. N. \& Semendyayev, K. A. 1985 Handbook of Mathematics. Verlag Harri Deutsch and Van Nostrand Reinhold Company, $973 \mathrm{pp}$.

Burns, A. G. \& Maslowe, S. A. 1983 Finite amplitude stability of a zonal shear flow. J. Atmos. Sci. 40, 3-9.

Carnevale, G. F. 1982 Statistical features of the evolution of two-dimensional turbulence. J. Fluid Mech. 122, 143-153.

Churilov, S. M. \& Shukhman, I. G. 1987 The nonlinear development of disturbances in a zonal shear flow. Geophys. Astrophys. Fluid Dyn. 38, 145-175.

DrITSChel, D. G. 1988 Nonlinear stability bounds for inviscid, two-dimensional, parallel or circular flows with monotonic vorticity, and the analogous three-dimensional quasigeostrophic flows. J. Fluid Mech. 191, 575-581.

FJøRтоғт, R. 1950 Application of integral theorems in deriving criteria of stability for laminar flows and for the baroclinic circular vortex. Geofys. Publr 17, no. 6, 52 pp.

Gradshteyn, I. S. \& Ryzhik, I. M. 1980 Table of Integrals, Series, and Products, Corrected and Enlarged Edition. Academic, $1160 \mathrm{pp}$.

HAYNES, P. H. 1988 Forced, dissipative generalizations of finite-amplitude wave-activity conservation relations for zonal and non-zonal basic flows. J. Atmos. Sci. 45, 2352-2362.

Holm, D. D., Marsden, J. E., Ratiu, T. \& Weinstein, A. 1985 Nonlinear stability of fluid and plasma equilibria. Phys. Rep. 123, 1-116.

Howard, L. N. 1972 Bounds on flow quantities. Ann. Rev. Fluid Mech. 4, 473-494.

Joseph, D. D. 1976 Stability of Fluid Motion, Part I. Springer, 282 pp.

Kraichnan, R. H. 1975 Statistical dynamics of two-dimensional flow. J. Fluid Mech. 67, $155-175$.

Kwon, H. J. \& Mak, M. 1988 On the equilibration in nonlinear barotropic instability. J. Atmos. Sci. 45, 294-308.

McIntyre, M. E. \& Shepherd, T. G. 1987 An exact local conservation theorem for finiteamplitude disturbances to non-parallel shear flows, with remarks on Hamiltonian structure and on Arnol'd's stability theorems. J. Fluid Mech. 181, 527-565.

Pierrenumbert, R. T. 1983 Bounds on the growth of perturbations to non-parallel steady flow on the barotropic beta-plane. J. Atmos. Sci. 40, 1207-1217.

RAYLEIGH, LORD 1880 On the stability, or instability, of certain fluid motions. Proc. Lond. Math. Soc. 11, 57-70. 
Salmon, R. 1982 Geostrophic turbulence. In Topics in Ocean Physics (ed. A. R. Osborne \& P. Malanotte-Rizzoli), Corso LXXX, pp. 30-78. Societa Italiana di Fisica, Bologna: NorthHolland.

Schoebert, M. R. \& Lindzen, R. S. 1984 A numerical simulation of barotropic instability. Part I: Wave-mean flow interaction. J. Atmos. Sci. 41, 1368-1379.

Shepherd, T. G. 1987 Non-ergodicity of inviscid two-dimensional flow on a beta-plane and on the surface of a rotating sphere. J. Fluid Mech. 184, 289-302.

ShePhERD, T. G. 1988 Nonlinear saturation of baroclinic instability. Part I : The two-layer model. J. Atmos. Sci. 45, 2014-2025.

Shukhman, I. G. 1987 Quasi-linear instability theory for a weakly supercritical zonal shear flow on the $\beta$-plane. Geophys. Astrophys. Fluid Dyn. 39, 105-118.

STUART, J.T. 1960 On the nonlinear mechanics of wave disturbances in stable and unstable parallel flows. Part 1. The basic behaviour in plane Poiseuille flow. J. Fluid Mech. 9, 353-370.

WATson, J. 1960 On the nonlinear mechanics of wave disturbances in stable and unstable parallel flows. Part 2. The development of a solution for plane Poiseuille flow and for plane Couette flow. J. Fluid Mech. 9, 371-389. 